\title{
Natural Product Trienomycin A is a STAT3 pathway inhibitor that exhibits potent in vitro and in vivo efficacy against pancreatic
}

\section{cancer}

\author{
Qiu-Rui He${ }^{1}$, Jiang-Jiang Tang ${ }^{1}$, Zhi-Fan Chen ${ }^{1}$, Yao Liu ${ }^{1}$, Huang Chen ${ }^{2}$, Ding Li $^{1}$, \\ Zhengfang $\mathrm{Yi}^{2}$, and Jin-Ming $\mathrm{Gao}^{1}$ \\ ${ }^{1}$ Northwest A\&F University \\ ${ }^{2}$ East China Normal University School of Life Sciences
}

October 20, 2020

\begin{abstract}
Background and Purpose: Pancreatic cancer is an exceptionally fatal disease. However, therapeutic drugs for pancreatic cancer have presented a serious shortage over the past few decades. Signal Transducer and Activator of Transcription-3 (STAT3) is persistently activated in many human cancers where it promotes tumor development and progression. Natural products serve as an inexhaustible source of anticancer drugs. Here, we identified the natural product Trienomycin A (TA), an ansamycin antibiotic, as a potential inhibitor of the STAT3 pathway with potent activity against pancreatic cancer. Experimental Approach: Utilizing the STAT3-luciferase (STAT3-luc) reporter system, we found that TA potently inhibits the transcriptional activity of STAT3. We subsequently investigated in vitro and in vivo inhibitory activity of TA against pancreatic cancer and its potential mechanism by using the molecular docking, SPR assay, MTS assay, colony formation assay, transwell migration/invasion assay, flow cytometric analysis, immunofluorescence staining, quantitative real-time PCR, western blotting, tumor xenograft model, H\&E staining and immunohistochemistry. Key Results: TA directly bound to STAT3 and inhibited STAT3 (Tyr705) phosphorylation, leading to the inhibition of the STAT3 pathway. TA significantly inhibited the colony formation, proliferation, migration and invasion of pancreatic cancer cell lines. TA dramatically blocked pancreatic tumor growth. More importantly, TA did not show obvious toxicity at the effective dose in mice. Conclusions and Implications: TA exhibits antineoplastic activity by suppressing the STAT3 activation in pancreatic cancer. TA could be a novel therapeutic candidate for pancreatic cancer by blocking the STAT3 pathway.
\end{abstract}

Natural Product Trienomycin A is a STAT3 pathway inhibitor that exhibits potent in vitro and in vivo efficacy against pancreatic cancer

Running title: Inhibiting STAT3 activity in pancreatic cancer

Qiu-Rui He ${ }^{1}$ Jiang-Jiang Tang ${ }^{1} \mid$ Zhi-Fan Chen ${ }^{1}$ Yao Liu ${ }^{1} \mid$ Huang Chen ${ }^{2} \mid$ Ding Li $^{1} \mid$ Zheng-Fang Yi $^{2}$ | Jin-Ming Gao ${ }^{1}$

${ }^{1}$ Shaanxi Key Laboratory of Natural Products \& Chemical Biology, College of Chemistry \& Pharmacy, Northwest A\&F University, Shaanxi, China

${ }^{2}$ Shanghai Key Laboratory of Regulatory Biology, Institute of Biomedical Sciences and School of Life Sciences, East China Normal University, Shanghai, China

\section{Correspondence}

Jin-Ming Gao, College of Chemistry \& Pharmacy, Northwest A\&F University, Yangling 712100, China. 


\section{Email:jinminggao@nwsuaf.edu.cn}

Zheng-Fang Yi, School of Life Sciences, East China Normal University, Shanghai 200241, China.

Email: zfyi@bio.ecnu.edu.cn

\section{Funding information}

National Natural Science Foundation of China, Grant/Award Numbers: 21877089, 21602177, and 21572182

Word count: 3,787 words, excluding abstract, methods, references, and figure legends.

Acknowledgements: This research was supported by the National Natural Science Foundation of China (21877089, 21602177, and 21572182). We thank Dr Zhengfang Yi at East China Normal University for the instructions.

Conflict of interest statement: The authors declare no conflicts of interest.

Data availability statement: The data that support the findings of this study are available from the corresponding author upon reasonable request. Some data may not be made available because of privacy or ethical restrictions.

Declaration of transparency and scientific rigour: This Declaration acknowledges that this paper adheres to the principles for transparent reporting and scientific rigour of preclinical research as stated in the $B J P$ guidelines for Design and Analysis, Immunoblotting and Immunochemistry, and Animal Experimentation, and as recommended by funding agencies, publishers and other organizations engaged with supporting research.

Abbreviations: MTS, 3-(4,5-Dimethylthiazol-2-yl)-5-(3-Carboxymethoxyphenyl)-2-(4-Sulfophenyl)-2HTetrazolium, Inner Salt; PCR, Polymerase Chain Reaction; DMSO, Dimethyl Sulfoxide; DMEM, Dulbecco's Modified Eagle's Medium; RPMI, Roswell Park Memorial Institute; IMDM, Iscove's Modified Dulbecco's Medium; HPLC, High Performance Liquid Chromatography; FBS, Fetal Bovine Serum; PBS, Phosphate Buffer Saline; FITC, Fluorescein Isothiocyanate; PI, Propidium Iodide; EDTA, Ethylenediaminetetraacetic Acid; AA, Amino Acids; NTA, Nitrilotriacetic Acid; RIPA, Radio Immunoprecipitation Assay; IL-6, Interleukin 6; RNA-seq, RNA sequencing; FC, Fold Change; KEGG, Kyoto Encyclopedia of Genes and Genomes; JAK, Janus kinase

\section{What is already known}

* Pancreatic cancer is an exceptionally lethal malignancy, while lacks effective therapeutic drugs.

* STAT3 is highly expressed and aberrantly activated in many human cancers.

\section{What this study adds}

* TA significantly inhibits the transcriptional activity of STAT3.

* TA exhibits potent efficacy against pancreatic cancer in vitroand in vivo .

\section{What is the clinical significance}

* STAT3 is a potential target for the prevention and treatment of pancreatic cancer.

* TA could be an innovative therapeutic candidate for pancreatic cancer.

\section{AUTHOR CONTRIBUTIONS}

J.-M.G. and Q.-R.H. designed the experiments. Q.-R.H. performed the experiments, analysed the data, and wrote the manuscript. J.-J.T. assisted with the animal experiments. Z.-F.C. and Y.L. prepared the natural compounds. H.C. aided in the screening of compounds. D.L. performed the molecular docking simulation. J.-M.G., J.-J.T., and Z.-F.Y. revised the manuscript. 


\section{ORCID}

Qiu-Rui He https://orcid.org/0000-0001-6823-3845

Jin-Ming Gao https://orcid.org/0000-0003-4801-6514

Background and Purpose: Pancreatic cancer is an exceptionally fatal disease. However, therapeutic drugs for pancreatic cancer have presented a serious shortage over the past few decades. Signal Transducer and Activator of Transcription-3 (STAT3) is persistently activated in many human cancers where it promotes tumor development and progression. Natural products serve as an inexhaustible source of anticancer drugs. Here, we identified the natural product Trienomycin A (TA), an ansamycin antibiotic, as a potential inhibitor of the STAT3 pathway with potent activity against pancreatic cancer.

Experimental Approach: Utilizing the STAT3-luciferase (STAT3-luc) reporter system, we found that TA potently inhibits the transcriptional activity of STAT3. We subsequently investigated in vitro and in vivo inhibitory activity of TA against pancreatic cancer and its potential mechanism by using the molecular docking, SPR assay, MTS assay, colony formation assay, transwell migration/invasion assay, flow cytometric analysis, immunofluorescence staining, quantitative real-time PCR, western blotting, tumor xenograft model, H\&E staining and immunohistochemistry.

Key Results: TA directly bound to STAT3 and inhibited STAT3 (Tyr705) phosphorylation, leading to the inhibition of the STAT3 pathway. TA significantly inhibited the colony formation, proliferation, migration and invasion of pancreatic cancer cell lines. TA dramatically blocked pancreatic tumor growth. More importantly, TA did not show obvious toxicity at the effective dose in mice.

Conclusions and Implications: TA exhibits antineoplastic activity by suppressing the STAT3 activation in pancreatic cancer. TA could be a novel therapeutic candidate for pancreatic cancer by blocking the STAT3 pathway.

Keywords: Trienomycin A; STAT3; Pancreatic cancer; Ansamycins; Natural product

\section{INTRODUCTION}

Pancreatic cancer is a highly lethal malignancy, which is characterized by the close parallel between cancer incidence and death rates, asymptomatic development, and an extremely poor prognosis (Kleeff et al., 2016; Aier, Semwal, Sharma, \& Varadwaj, 2019). According to global cancer statistics, pancreatic cancer is the seventh leading cause of cancer-associated mortality worldwide in 2018 (Bray et al., 2018). Due to the rapid cancer progression without specific symptoms, most patients with pancreatic cancer are diagnosed at advanced stages. The 5-year relative survival rate in patients diagnosed with the disease remains as low as $9 \%$ in the United States (Siegel, Miller, \& Jemal, 2020). Therefore, pancreatic cancer is commonly fatal. Pancreatic cancer is predicted to become the second leading death cause from cancer in the United States by 2030 (Rahib et al., 2014). However, over the past few decades, therapeutic drugs for pancreatic cancer have yielded very limited success in clinical trials (Roth, Cardin, \& Berlin, 2020; Kamisawa, Wood, Itoi, \& Takaori, 2016). Moreover, drug resistance is a serious problem in the treatment of pancreatic cancer (Binenbaum, Na'ara, \& Gil, 2015). Thus, development of effective targeted agents for treating pancreatic cancer is urgently needed.

STAT3 is a pro-oncogenic transcription factor, and is regarded as a therapeutic target in numerous human cancers, including lung cancer, colorectal cancer, breast cancer, prostate cancer, ovarian cancer and so on (Dutta, Sabri, Li, \& Li, 2015; Banerjee \& Resat, 2016; Ouédraogo et al., 2017; Liang et al., 2020). STAT3 regulates the expression of its downstream target genes related to cell proliferation, survival, migration, invasion, epithelial-mesenchymal transition, tumor angiogenesis, the Warburg effect, cancer stem cell properties, and immune evasion closely connected with cancer progression and malignancy (Carpenter \& Lo, 2014; Kamran, Patil, \& Gude, 2013; Wendt, Balanis, Carlin, \& Schiemann, 2014; Gao et al., 2017; Demaria et al., 2010; Galoczova, Coates, \& Vojtesek, 2018; Wang et al., 2004). Persistently activated STAT3 also mediates cancer-associated inflammation that can initiate and promote malignant transformation, and 
genetic alterations in pre-neoplastic and malignant cells can also induce and maintain a tumor-promoting inflammatory microenvironment that further facilitates tumorigenesis and malignant progression (Fan, Mao, \& Yang, 2013; Yu, Lee, Herrmann, Buettner, \& Jove, 2014; Yu, Pardoll, \& Jove, 2009). In addition to the tumor cells, STAT3, which is a converging point of numerous oncogenic pathways, is also constitutively activated in tumor-infiltrated immune cells, resulting in suppression of immune mediators and promotion of immunosuppressive factors (Wang, Shen, Wang, Shen, \& Zhou, 2018; Yu, Kortylewski, \& Pardoll, 2007). In addition, accumulating evidence also indicates that feedback activation of STAT3 plays a critical role in mediating the drug resistance that often follows the targeted cancer therapies and conventional chemotherapies (Lee et al., 2017; Zhao et al., 2016). Taken together, inhibiting STAT3 activation is an attractive and promising approach for cancer prevention, therapy and drug sensitization (Johnson, O'Keefe, \& Grandis, 2018; Furtek, Backos, Matheson, \& Reigan, 2016). Overexpression and hyperactivation of the oncoprotein STAT3 frequently occur in pancreatic cancer (Scholz et al., 2003; Fofaria \& Srivastava, 2015; He et al., 2018; Wei et al., 2003). STAT3 is also a major mediator in KRAS -driven pancreatic cancer that lacks clinically actionable targets (Li, Grivennikov, \& Karin, 2011; D'Amico et al., 2018). Hence, inhibiting aberrantly activated STAT3 is a promising strategy for pancreatic cancer therapy.

To identify natural compounds that block the transcriptional activity of STAT3, we utilized the STAT3luciferase (STAT3-luc) reporter system containing STAT3-binding elements to screen potentially active compounds from our in-house natural product library including more than 1000 compounds and identified a natural compound termed Trienomycin A (TA), a secondary metabolite produced by the moss-soil-derived actinomyceteStreptomyces cacaoi subsp. asoensis H2S5, that has anti-neuroinflammatory and anti-tumor activities (Tang et al., 2018; Liu et al., 2020). However, very little is known about the impact of TA on pancreatic cancer, and the underlying molecular mechanisms of TA are also unclear. In this study, we found that TA memorably repressed the transcriptional activity of STAT3. We further demonstrated TA inhibited the phosphorylation of tyrosine at the 705 site of STAT3 and the protein expression of downstream target genes that related to cell proliferation, growth, migration and invasion, by binding to STAT3 protein. Furthermore, we found that TA significantly reduced the growth of pancreatic tumor in vivo, and that TA had no obvious toxic effects on the normal tissues (heart, liver, spleen, lung and kidney) of mice. Collectively, our data give the first evidence that TA is a potential STAT3 pathway inhibitor with significant activity against pancreatic cancer, and it is worthwhile to be further studied.

\section{METHODS}

\subsection{Cell lines and reagents}

Human pancreatic cancer cells, PANC-1 (RRID:CVCL_0480), BxPC-3 (RRID:CVCL_0186), MIA PaCa-2 (RRID:CVCL_0428), CFPAC-1 (RRID:CVCL_1119), AsPC-1 (RRID:CVCL_0152), SW1990 (RRID:CVCL_1723), Capan-2 (RRID:CVCL_0026), and HPAC (RRID:CVCL_3517), human prostate cancer cells, PC-3 (RRID:CVCL_0035) and PC-3M (RRID:CVCL_9555), human breast cancer cells, MDA-MB-231 (RRID:CVCL_0062), and other normal cells, 293T (RRID:CVCL_0063), HaCaT (RRID:CVCL_0038), HAF (RRID:CVCL_ZG36), and C2C12 (RRID:CVCL_0188), were purchased from Cell Bank of the Chinese Academy of Sciences (Shanghai, China) or the American Type Culture Collection (Manassas, VA, USA). PANC-1, HPAC, HaCaT, HAF, and C2C12 cells were cultured in DMEM (Gibco) supplemented with 10\% FBS (Gibco) and $1 \%$ penicillin/streptomycin (Gibco). MIAPaCa-2 cells were maintained in DMEM supplemented with $10 \%$ FBS, $2.5 \%$ horse serum (Gibco), $1 \%$ sodium pyruvate $100 \mathrm{mM}$ solution (Invitrogen) and 1\% penicillin/streptomycin. AsPC-1, BxPC-3, Capan-2, and PC-3M cells were cultured in RPMI 1640 (Gibco) supplemented with $10 \% \mathrm{FBS}$ and $1 \%$ penicillin/streptomycin. CFPAC-1 cells were grown in IMDM (Gibco) supplemented with 10\% FBS and 1\% penicillin/streptomycin. SW1990 and MDA-MB-231 cells were cultured in L-15 (Gibco) supplemented with 10\% FBS and 1\% penicillin/streptomycin. PC-3 cells were maintained in DMEM/F12 (Hyclone) medium supplemented with 10\% FBS and 1\% penicillin/streptomycin. 293T cells were cultured in DMEM supplemented with $10 \%$ FBS. All cell lines were maintained at $37{ }^{\circ} \mathrm{C}$ in a humidified incubator containing $5 \% \mathrm{CO}_{2}$. Trienomycin A (purity: $>95 \%$ by HPLC) was isolated and purified by ourselves as previously described (Tang et al., 2018). TA was dissolved in DMSO as a $10 \mathrm{mM}$ stock 
solution and stored at $-20{ }^{\circ} \mathrm{C}$. Gemcitabine hydrochloride was purchased from MedChemExpress (Shanghai, China). All STAT3 inhibitors (BP-1-102 and C188-9, dissolved in DMSO) were purchased from Topscience (Shanghai, China). Recombinant human STAT3 (127-722 AA) protein was kindly provided by Dr Zhengfang Yi (Institute of Biomedical Sciences and School of Life Sciences, East China Normal University, China).

\subsection{Dual luciferase screening assay}

Both Firefly and Renilla luciferase activities were measured using the Dual-Luciferase Reporter Assay System (Promega, Madison, WI, USA) according to the manufacturer's instructions. 293T cells were plated in 24-well culture plates and co-transfected transiently with the STAT3-luc and Renilla plasmids using Lipofectamine 2000 (Invitrogen, Carlsbad, CA, USA). After incubation for $24 \mathrm{~h}$, the transfected cells were stimulated with recombinant human interleukin-6 (Roche) and treated with TA $(0,0.1$, and $1 \mu \mathrm{M})$ for $12 \mathrm{~h}$. Afterwards, the cell lysates were prepared and the activities ofFirefly and Renilla luciferases were quantified using a Veritas Microplate Luminometer (Turner Biosystems, Sunnyvale, CA, USA). The data were expressed as the ratio of Firefly to Renillaluciferase activity.

\subsection{Cell viability assay}

Cell viability was measured with MTS Assay Kit (Promega, Madison, WI, USA) according to the manufacturer's instructions. Pancreatic cancer cell lines were seeded in 96-well plates at 2,000-5,000 cells per well (depending on the cell lines used). After incubation for $24 \mathrm{~h}$, the cells were treated with TA at concentrations ranging from 0 to $10 \mu \mathrm{M}$ for $48 \mathrm{~h}$ or $72 \mathrm{~h}$. Then, $20 \mu \mathrm{L}$ of MTS solution was added directly into each well, and the 96 -well plates were incubated at $37{ }^{\circ} \mathrm{C}$ in a humidified incubator with $5 \% \mathrm{CO}_{2}$ for $1-2 \mathrm{~h}$. The absorbance of each well at $490 \mathrm{~nm}$ was recorded using a 96-well plate reader (Bio-Rad, Hercules, CA, USA).

\subsection{Colony formation assay}

The clonogenic growth of pancreatic cancer cells was evaluated by plate colony formation assay. The cells were plated into six-well plates at 300-500 cells per well (depending on the cell lines used). After incubation for $24 \mathrm{~h}$, the cells were treated with various concentrations of TA. The plates were incubated at $37 \operatorname{degC}$ in a humidified incubator containing $5 \% \mathrm{CO}_{2}$ for 7 days. The colonies were washed twice with cold PBS and fixed with $4 \%$ paraformaldehyde, then stained with $0.1 \%$ crystal violet. The images of stained colonies were captured using a digital camera (Nikon, Tokyo, Japan).

\subsection{Transwell migration/invasion assay}

The migration and invasion abilities of pancreatic cancer cells were determined by using Transwell Boyden Chambers with 8-um pores (Corning Inc., Lowell, MA, USA). The upper Transwell chambers were uncoated for cell migration assay or pre-coated with $50 \mu \mathrm{L}$ of Matrigel (1:4 mixed with PBS; BD Biosciences, San Jose, CA, USA) for cell invasion assay. The cells were precultured in serum-free medium for $12 \mathrm{~h}$. Next, the cells were harvested and resuspended in serum-free medium containing serial concentrations of TA, and then plated on the upper chambers at an appropriate density. At the same time, complete medium containing $10 \%$ FBS was added to the lower chambers. After an incubation of $48 \mathrm{~h}$, the tumor cells on the upper side of the filters were wiped off with cotton swabs. The tumor cells on the lower surface of the membrane were then fixed with $4 \%$ paraformaldehyde and stained with $0.1 \%$ crystal violet. The images of stained tumor cells were acquired using an inverted microscope (Olympus, Tokyo, Japan).

\subsection{Flow cytometric assay}

For cell cycle analysis, pancreatic cancer cells were seeded in $6 \mathrm{~cm}$ dishes and incubated overnight. The cells were treated with indicated concentrations of TA for $24 \mathrm{~h}$. The treated cells were collected and washed twice with cold PBS. Following that, the cells were fixed with pre-cold $70 \%$ ethanol at $4{ }^{\circ} \mathrm{C}$ overnight. The fixed cells were washed one time with PBS and then resuspended in PBS containing PI $(50 \mu \mathrm{g} / \mathrm{mL})$ and DNasefree RNase $(100 \mu \mathrm{g} / \mathrm{mL})$, and incubated at room temperature for $30 \mathrm{~min}$ in the dark. Subsequently, the cell cycle analysis was performed using the FACSCalibur flow cytometer (BD Biosciences, CA, USA). The 
percentage of cells in different cell cycle phases was determined by the ModFit LT software (Verity Software House, Topsham, ME, USA).

The apoptosis rate of tumor cells was detected by flow cytometry with Annexin V-FITC Apoptosis Detection Kit (BD Biosciences, San Jose, CA), following the manufacturer's instructions. Pancreatic cancer cells were cultured in six-well plates. After reaching 70-80\% confluence, the cells were treated with indicated concentrations of TA for $48 \mathrm{~h}$. After that, the cells were harvested, washed twice with cold PBS, and resuspended in $100 \mu \mathrm{L}$ of $1 \times$ binding buffer. The cells were stained with $5 \mu \mathrm{L}$ of Annexin V-fluorescein isothiocyanate and $5 \mu \mathrm{L}$ of PI at room temperature for $15 \mathrm{~min}$ in the dark. Finally, $400 \mu \mathrm{L}$ of $1 \times$ binding buffer was added to the cell suspension. The apoptosis rate was detected using the FACSCalibur flow cytometer (BD Biosciences, CA, USA). The data were analyzed with the FlowJo software (TreeStar, Inc., Ashland, OR, USA).

\subsection{Molecular docking}

In order to predict the possible interaction patterns between natural compounds and STAT3, molecular docking studies were carried out using Autodock 4.2 software (The Scripps Research Institute, La Jolla, CA, USA). The preparations related to Autodock docking were performed using the Autodock Tools (ADT). The three-dimensional (3D) crystal structure of STAT3 (PDB code: 1BG1) was retrieved from the RCSB Protein Data Bank (PDB; http://www.rcsb.org/pdb/). The standard 3D structures of natural compounds were constructed by using the "SKETCH" option function in SYBYL-X 2.1 (Tripos International, St. Louis, MO, USA). The Lamarckian genetic algorithm (LGA) was used to implement the molecular docking. The docking parameters were set as follows: population size, 150; number of generations, 270 000; number of evaluations, 25000 000; number of top individuals that automatically survive, 20; and number of docking runs, 40. The default values of the remaining parameters were used.

\subsection{Surface plasmon resonance assay}

The binding affinity of natural compounds to STAT3 (127-722 AA) was evaluated by Surface Plasmon Resonance (SPR) assay using a Biacore T200 instrument (GE Healthcare, Piscataway, NJ, USA). After conditioning with $350 \mathrm{mM}$ EDTA and $50 \mathrm{mM} \mathrm{NaOH}, 50 \mu \mathrm{g} / \mathrm{mL}$ of STAT3 protein was immobilized onto an NTA sensor chip at a flow rate of $10 \mu \mathrm{L} / \mathrm{min}$ in PBS containing $0.05 \%$ (v/v) Tween-20. The serial concentrations of natural compounds were injected into the flow system and analyzed, respectively. All measurements were performed in PBS with $0.05 \%$ (v/v) Tween-20, $\mathrm{pH} 7.4$, at $25{ }^{\circ} \mathrm{C}$. The STAT3 protein and natural compounds were allowed to associate for $90 \mathrm{~s}$ and then dissociate for $120 \mathrm{~s}$. After dissociation, the sensor chip was washed with running buffer and regenerated with $350 \mathrm{mM}$ EDTA and $50 \mathrm{mM} \mathrm{NaOH}$. To avoid or reduce the influences of the bulk refractive index changes, data drift and injection noise, we performed the double reference subtractions prior to analysis. The binding affinity was measured by global fitting to a 1:1 Langmuir model using the Biacore Evaluation software (GE Healthcare).

\subsection{Quantitative Real-Time PCR}

Total RNA was extracted from tumor cells or tissues using TRIzol reagent (Takara, Tokyo, Japan) according to the manufacturer's protocol. The concentration and purity of total RNA were determined using a Nanodrop ND-1000 spectrophotomer (Thermo Fisher Scientific, Wilmington, DE, USA), with an A260/A280 ratio between 1.8 and 2.0. Complementary DNA (cDNA) was synthesized using cDNA Reverse Transcription Kit (Takara, Tokyo, Japan). Quantitative Real-Time PCR (qRTPCR) was performed using SYBR Premix Ex Taq (Takara, Tokyo, Japan) according to the manufacturer's instructions in a QuantStudio(r)3 Real-Time PCR System (Applied Biosystems, Foster City, CA, USA). The expression levels of STAT3 downstream target genes were calculated using the $2^{-C T}$ method. GAPDH was used as an internal reference for the relative quantifications. For the total RNA extraction of tissues, a small portion of tumor tissues was removed, frozen in liquid nitrogen, and homogenized in the reagent described above with $5 \mathrm{~mm}$ stainless steel beads (QIAGEN Inc., Valencia, CA, USA) in the TissueLyser system (QIAGEN Inc.). The remaining procedures were then performed as described above. The primers used were as follows: VEGF forward, 5'CGAGATAGAGTACATCTTCAAGC-3', and 
reverse, 5'-TTGATCCGCATGATCTGCATGG-3'; IL6 forward, 5'-AAATGCCAGCCTGCTGACGAAC3', and reverse, 5'-AACAACAATCTGAGGTGCCCATGCTA-3'; CASP3 forward, 5'AACTGGACTGTGGCATTGAG-3', and reverse, 5'-ACAAAGCGACTGGATGAACC-3'; BCL2 forward, 5'-AGACCGAAGTCCGCAGAACC-3', and reverse, 5'-GAGACCACACTGCCCTGTTG-3'; ICAM1 forward, 5'-TCAAAAGTCATCCTGCCCCG-3', and reverse, 5'-TGCTCAGTTCATACACCTTCCG3'; CCND1 forward, 5'-TCTACACCGACAACTCCATCC-3', and reverse, 5'TTCCACTTGAGCTTGTTCACC-3';MMP9 forward, 5'-AGACGGGTATCCCTTCGACG3', and reverse, 5'-AAACCGAGTTGGAACCACGAC-3'; $M M P^{2}$ forward, 5'GCCCCAGACAGGTGATCTTG-3', and reverse, 5'-GCTTGCGAGGGAAGAAGTTGT-3'; $M Y C$ forward, 5'-GGCTCCTGGCAAAAGGTCA-3', and reverse, 5'-CTGCGTAGTTGTGCTGATGT-3'; TXNIP forward, 5'-TCGTGTCAAAGCCGTTAGGA-3', and reverse, 5'-ATTCTCACCTGTTGGCTGGT-3';NOP56 forward, 5'-ACAGCTATTCCCGTGCCAAA-3', and reverse, 5'-CCACTCCCTGACACGCATAG3'; MFSD2A forward, 5'-AGCTTTGCTATGCACTTGGGG-3', and reverse, 5'TCATCCTTCTGAGCCACATCCA-3';SAT1 forward, 5'-CTCCGGAAGGACACAGCATT-3', and reverse, 5'-TGATCCTATGCCAAAGCCTCT-3'; $F 3$ forward, 5'-CAGGAAAGAAAACAGCCAAAACA-3', and reverse, 5'-TACCGGGCTGTCTGTACTCT-3';CLDN1 forward, 5'-ATTTCTTCTTGCAGGTCTGGCT3', and reverse, 5'-GGGGACAGGAACAGCAAAGT-3'; SLC12A3 forward, 5'CGCACCTTTGGCTACAACAC-3', and reverse, 5'-GTGTCTGCCTTCCTGCTTGA-3';HSPA8 forward, 5'-GCCTACACCCCAGCAACCAT-3', and reverse, 5'-TTGGAGTGGTTCGGTTTCCC-3'; TRIM31 forward, 5'-CGGAAGCGGGGAAACACTAT-3', and reverse, 5'-CTGAAACTCTTCACTTCTGCAC-3'; MN1 forward, 5'-GGTACATGCCCGCTGACAA-3', and reverse, 5'-GAGGTCGTGGGCTTCTTTGCT-3'; GAPDH forward, 5'-CGACAGTCAGCCGCATCTT-3', and reverse, 5'-CCGTTGACTCCGACCTTCA-3'.

\subsection{RNA-Seq and bioinformatics analysis}

Pancreatic cancer cells were plated in six-well plates. After overnight incubation, the cells were treated with different concentrations of TA for $24 \mathrm{~h}$. Total RNA was prepared as mentioned above. A total amount of 2 $\mu \mathrm{g}$ RNA per sample was used as input material for the RNA sample preparations. Sequencing libraries were constructed with the VAHTS mRNA-seq v2 Library Prep Kit for Illumina (Vazyme Biotech Co., Ltd., Nanjing, China) following the manufacturer's instructions, and index codes were added to attribute sequences to each sample. Transcriptomic sequencing was performed by using the Illumina NovaSeq 6000 platform (Illumina, Inc., San Diego, CA, USA) with three biological replicates, and 150-bp paired-end reads were generated. The differentially expressed genes (DEGs) between treated and untreated groups were identified by using the DEGSeq R package (1.20.0). Gene ontology (GO) analysis of DEGs was performed using the Gene Ontology Annotation (GOA) database (http://www.ebi.ac.uk/GOA). KEGG pathway analysis of DEGs was conducted using the KEGG Automatic Annotation Server (KAAS; http://www.genome.jp/tools/kaas/).

\subsection{Western blot analysis}

Pancreatic cancer cells were seeded in six-well plates and incubated overnight. The cells were subsequently treated with different concentrations of TA for $24 \mathrm{~h}$. The cells were harvested and lysed in the RIPA buffer (Beyotime Biotechnology, Shanghai, China) containing protease and phosphatase inhibitor cocktail (Thermo Fisher Scientific) on ice for 20 minutes. After centrifugation for 20 min at 12 $000 \mathrm{rpm}$ under $4^{\circ} \mathrm{C}$, the supernatants were collected. The protein concentration was then quantified by the Pierce BCA Protein Assay Kit (Thermo Fisher Scientific Inc., San Jose, CA, USA). Equal amounts of protein $(20-30 \mu \mathrm{g})$ were separated by $12 \%$ sodium dodecyl sulfate-polyacrylamide gel electrophoresis (SDS-PAGE) and then transferred onto nitrocellulose membranes (Bio-Rad, Hercules, CA, USA). The membranes were blocked with Tris-buffered saline with 0.05\% Tween-20 (TBST) supplemented with 5\% nonfat dry milk for $1 \mathrm{~h}$ at room temperature. After blocking, the membranes were incubated with specific primary antibodies overnight at $4^{\circ} \mathrm{C}$. After washing three times with TBST, the membranes were incubated with a IRDye 800CW secondary antibody (LI-COR Biosciences, Lincoln, NE, USA; RRID:AB_621843; 1:10 000) for $1 \mathrm{~h}$ at room temperature. Following washing three times with TBST, the membranes were scanned and visualized by using the Odyssey Infrared Imaging System (LI-COR Biosciences, Lincoln, NE, USA). For the protein 
extraction of tissues, a small piece of tumor tissues was removed, frozen in liquid nitrogen, and homogenized in the lysis buffer described above with $5 \mathrm{~mm}$ stainless steel beads (QIAGEN Inc., Valencia, CA, USA) in the TissueLyser system (QIAGEN Inc.). The remaining operations were then performed as described above. Primary antibodies including p-STAT3 (Tyr705) (RRID:AB_2491009), p-STAT3 (Ser727) (RRID:AB_331589), STAT3 (RRID:AB_2629499), c-Myc (RRID:AB_1903938), Cyclin D1 (RRID:AB_2827374), Survivin (RRID:AB_2063948), Bcl2 (RRID:AB_1903909), ICAM-1 (RRID:AB_2280018), MMP-9 (RRID:AB_2798289) and GAPDH (RRID:AB_10622025) were purchased from Cell Signaling Technology (Beverly, MA, USA). The immune-related procedures used comply with the recommendations made by theBritish Journal of Pharmacology (Alexander et al., 2018).

\subsection{Subcutaneous tumor xenograft models}

All protocols involving mice in this study were conducted in accordance with the principles and procedures approved by the Animal Ethics Committee of Northwest A\&F University. Animal studies are reported in compliance with the ARRIVE guidelines (Kilkenny, Browne, Cuthill, Emerson, \& Altman, 2010; McGrath \& Lilley, 2015) and with the recommendations made by the British Journal of Pharmacology. Male BALB/c nude mice (6 to 8 weeks old) were purchased from Sino-British Sippr/BK Laboratory Animal Ltd. (Shanghai, China). The mice were maintained under specific pathogen-free conditions. Five mice were kept in one breeding cage. PANC- 1 cells $\left(1 \times 10^{6}\right.$ cells suspended in $0.1 \mathrm{~mL}$ sterile PBS/animal $)$ were subcutaneously injected into the right flanks of mice with a $1 \mathrm{~mL}$ sterile disposable plastic syringe. When the tumors reached an average volume of $100 \mathrm{~mm}^{3}$, the mice were randomly divided into five groups with five mice in each group. The five groups were treated with solvent (PBS with 10\% methanol and 10\% Tween-80), gemcitabine hydrochloride $(80 \mathrm{mg} / \mathrm{kg} / 3 \mathrm{~d})$, TA $(10 \mathrm{mg} / \mathrm{kg} / \mathrm{d})$, TA $(20 \mathrm{mg} / \mathrm{kg} / \mathrm{d})$, and TA $(40 \mathrm{mg} / \mathrm{kg} / \mathrm{d})$ by intraperitoneal injection 15 days, respectively. Tumor volume and body weight of the tumor-bearing nude mice were monitored once every three days. The tumor volume was calculated using the following formula: tumor volume $(\mathrm{V})=$ length $\times$ width $\times$ width $\times 0.52$. At the end of the experiment, the tumor-bearing nude mice were sacrificed by cervical dislocation. The tumors were separated from the surrounding muscles and dermis, weighed, and photographed.

\subsection{Histology and immunohistochemistry}

Tissue samples were collected to perform histological assessments. Tumor tissues or mouse tissues were immediately fixed with $10 \%$ neutral buffered formaldehyde. After fixation for at least $24 \mathrm{~h}$, the tissues were progressively dehydrated in a series of graded ethyl alcohol solutions (70,80, 95 and 100\%, v/v), and embedded into paraffin blocks. For pathological examination, tissue sections $(4 \mu \mathrm{m}$ thickness $)$ were cut from paraffin blocks of heart, lung, spleen, liver, kidney, and tumor. Subsequently, the sections were stained with hematoxylin and eosin (H\&E) to indicate nucleus and cytoplasm, respectively. For immunohistochemical staining, tissue sections were prepared as described above. The sections were immersed in a Tris/EDTA buffer ( $\mathrm{pH}$ 9.0; Servicebio, Wuhan, China) and were heated for $15 \mathrm{~min}$ in a microwave oven. Following antigen retrieval, the sections were incubated with $3 \%$ hydrogen peroxide for 10 min to block endogenous peroxidase activity. After the inactivation of peroxidase, the sections were blocked with $3 \%$ BSA for 30 min at room temperature. The sections were incubated with primary antibodies at $4{ }^{\circ} \mathrm{C}$ overnight, washed three times with TBST, and incubated with the corresponding HRP-conjugated secondary antibody (Invitrogen Corporation, Carlsbad, CA, USA; RRID:AB_2533965; 1:2000) for 50 min at room temperature in the dark. After washing three times with TBST, 3,3'-diaminobenzidine (DAB) was dropped as a chromogen, forming a brown-colored precipitate at the antigenic sites. Finally, the sections were counterstained with hematoxylin. All stained slides were dried, mounted with neutral gum, and examined under a light microscope (Olympus, Tokyo, Japan). Primary antibodies including PCNA (RRID:AB_303394), Ki67 (RRID:AB_443209), p-STAT3 (Tyr705) (RRID:AB_1658549), c-Myc (RRID:AB_731658), MMP-9 (RRID:AB_1310463), Survivin (RRID:AB_304564) and ICAM-1 (RRID:AB_870702) were purchased from Abcam (Cambridge, UK). The immune-related procedures used comply with the recommendations made by the British Journal of Pharmacology (Alexander et al., 2018).

\subsection{Tissue immunofluorescence staining}


For tumor immunofluorescence staining, tissue sections were prepared as described above. The sections were immersed in a Tris/EDTA buffer (pH 8.0; Servicebio, Wuhan, China) and were heated for 15 min in a microwave oven. After antigen retrieval, the sections were circled with a PAP pen (Electron Microscopy Sciences, Fort Washington, PA, USA) to reduce reagent waste. The sections were incubated with $3 \%$ BSA for $30 \mathrm{~min}$ at room temperature. Afterwards, the sections were incubated with primary antibody at 4 $\operatorname{degC}$ overnight, washed three times with TBST, and incubated with the corresponding FITC-conjugated secondary antibody (Invitrogen Corporation, Carlsbad, CA, USA; RRID:AB_2535643; 1:500) for 50 min at room temperature in the dark. After washing with TBST for three times, the sections were incubated with tissue autofluorescence quencher for 5 min. Ultimately, the sections were stained with DAPI for 10 min. All stained slides were mounted with Vectashield mounting medium (Vector Laboratories, Burlingame, CA, USA). The fluorescence images were captured by a fluorescence microscope (Leica, Wetzlar, Germany).

\subsection{Pharmacokinetic study}

All animal experimental protocols were carried out strictly in accordance with the principles and procedures approved by the Animal Ethics Committee of Northwest A\&F University. Animal studies are reported in compliance with the ARRIVE guidelines (Kilkenny, Browne, Cuthill, Emerson, \& Altman, 2010; McGrath \& Lilley, 2015) and with the recommendations made by the British Journal of Pharmacology . Male SpragueDawley rats (200-220 g) were purchased from Sino-British Sippr/BK Laboratory Animal Ltd. (Shanghai, China). Rats were fasted but allowed free access to water for $12 \mathrm{~h}$ prior to the pharmacokinetic study. The rats were randomized into two groups for intravenous or intraperitoneal injection, the dosages of which were set at 20 and $40 \mathrm{mg} / \mathrm{kg}$, respectively. Each group contained five or six rats. Blood samples were collected from the orbital plexus into heparinized centrifuge tubes at 5, 10, 20,40,60,120, 240, 360, 720, and $1440 \mathrm{~min}$ after the administration. Then the whole blood was centrifuged at $4000 \mathrm{x} \mathrm{g}$ for $10 \mathrm{~min}$ at $4 \operatorname{deg}$ C. The obtained plasma was frozen and stored at $-80 \operatorname{deg} \mathrm{C}$ until further analysis. Pharmacokinetic parameters were calculated by WinNonlin software (Pharsight Corporation, Princeton, NJ, USA) based on non-compartmental method. Mean plasma concentration-time curves of TA in rats were plotted by GraphPad Prism 5 (GraphPad software Inc., CA, USA).

\subsection{Data and statistical analysis}

The data and statistical analysis comply with the recommendations of theBritish Journal of Pharmacology on experimental design and analysis in pharmacology (Curtis et al., 2018). All data in the text, tables, and figures are presented as means +- SD or means +- SEM. Statistical analysis was performed using GraphPad Prism 5 (GraphPad Software, Inc., La Jolla, CA, USA, RRID:SCR_002798). Statistical significance was determined by using two-way ANOVA or unpaired two-tailed Student's $t$-test. $P$ values $<0.05$ were considered statistically significant. Representative data are presented as raw data. Unless indicated otherwise, five or more independent experiments were performed. Blinding or randomization was undertaken in this study.

\section{RESULTS}

\subsection{TA blocks the STAT3 signaling pathway}

To identify natural compounds that inhibit the transcriptional activity of STAT3, we used a luciferase reporter assay to screen natural compounds as potential STAT3 pathway inhibitors. 293T cells were stimulated with IL-6 to activate the STAT3 signaling pathway. After incubation with these natural compounds for $12 \mathrm{~h}$, the transfected cells were collected and STAT3 transcriptional activity was detected by dual luciferase assay. We identified a natural product, Trienomycin A (TA), that potently reduced the transcriptional activity of STAT3. As shown in Figure 1b, TA dose-dependently inhibited the IL-6-induced transcriptional activity of STAT3.

As mentioned above, STAT3 has been considered as a potential anticancer target. Accordingly, we next investigated whether TA interacted with STAT3. We performed the molecular docking and binding free energy $\left(\Delta \mathrm{G}_{\mathrm{bind}}\right)$ calculation using the X-ray crystal structure of the STAT3 $\beta /$ DNA complex (PDB:1BG1), 
and identified a potential binding site on the surface of STAT3 that could reasonably accommodate TA binding. As shown in Figure 1c, the oxygen atom of phenolic hydroxyl group on C-22 of TA forms hydrogen bond with the hydrogen atom of Asn485 side-chain amide. The oxygen atom of methoxy group on C-3 of TA forms hydrogen bond with the hydrogen atom of Lys488 side-chain amine. The substituent group on C-11 of TA is very important for the combination with STAT3, and the two oxygen atoms of its ester group form hydrogen bonds with the hydrogen atoms of Lys244 side-chain amine, and the oxygen atom (carbonyl oxygen atom on C-30) of its amide group forms hydrogen bond with the hydrogen atom of His 457 framework amide. Moreover, we found that TA could fit to the hot spots of STAT3 protein with a binding energy of $-9.32 \mathrm{kcal} / \mathrm{mol}$. Probably, TA directly interacted with STAT3, inducing a change in the conformation of the protein, resulting in the inhibitory effects on the STAT3 signaling pathway.

To further validate the direct interaction of TA with STAT3, the binding affinity of TA with STAT3 was detected by the SPR assay. As shown in Figure 1d, we observed that TA interacted with STAT3 protein with an equilibrium dissociation constant (KD) of $18.00 \mu \mathrm{mol} / \mathrm{L}$. In addition, the structural analogues of TA could also bind directly to STAT3 with KD values range from $4.42 \mu \mathrm{mol} / \mathrm{L}$ to $17.78 \mu \mathrm{mol} / \mathrm{L}$ (Figure S1a). As shown in Figure S1b, the STAT3 inhibitors, C188-9 and BP-1-102, bound to STAT3 with a KD value of $3.71 \mu \mathrm{mol} / \mathrm{L}$ and $0.84 \mu \mathrm{mol} / \mathrm{L}$, which were approximately equal to values reported in literature, respectively (Bharadwaj et al., 2016; Zhang et al., 2012). To sum up, all these data indicated that TA most probably inhibited STAT3 signaling pathway by targeting STAT3.

We next examined whether TA could suppress the STAT3 pathway in pancreatic cancer. Firstly, we searched the expression of p-STAT3 (Tyr705) protein in various cancer cell lines by using the Cancer Cell Line Encyclopedia (CCLE). It was intriguing to find that p-STAT3 (Tyr705) protein was highly expressed in pancreatic cancer compared with most other cancer cell lines such as gastric cancer, breast cancer, ovarian cancer and so on (Figure S1c). In order to further detect the expression and activation of STAT3, the expression levels of total STAT3 and p-STAT3 (Tyr705 and Ser727) were evaluated in human pancreatic cancer cell lines (PANC-1, BxPC-3, MIA PaCa-2, Capan-2, CFPAC-1, AsPC-1, SW1990, and HPAC). As demonstrated in Figure 1e, the PANC-1, CFPAC-1, Capan-2, BxPC-3, and HPAC cell lines showed higher levels of p-STAT3 (Tyr705) protein compared with the rest. Based on these results, the PANC-1, CFPAC-1, Capan-2, and BxPC-3 cell lines were selected for subsequent experiments.

Subsequently, we investigated the effect of TA on the expression levels of total STAT3, p-STAT3 (Tyr705 and Ser727), and STAT3-regulated proteins in human pancreatic cancer cell lines. As shown in Figures 1f and S1d, TA remarkably inhibited p-STAT3 (Tyr705) expression levels in a dose-dependent manner, while had no concentration-dependent suppressive effect on p-STAT3 (Ser727) and total STAT3 expression levels. TA also significantly down-regulated the protein expression of STAT3 downstream target genes (Cyclin D1, Survivin, and ICAM-1) in a dose-dependent way. Taken together, these results demonstrated that TA substantially inhibited the STAT3 signaling pathway.

\subsection{TA inhibits the proliferation and growth of pancreatic cancer cells}

We subsequently examined whether the structural analogues of TA affected the proliferation of pancreatic cancer cells (PANC-1, BxPC-3, and Capan-2). Using the MTS assay, we confirmed that the structural analogues of TA displayed differential inhibitory effects on the cell proliferation of pancreatic cancer (Figure $2 \mathrm{~b})$. Among these compounds, TA showed the strongest anti-proliferation activity against pancreatic cancer cells. The preliminary structure-activity relationship of these compounds showed that the position of the side chain attached onansa ring played an important role in the anticancer activity, and the existence of a cyclohexyl group significantly improved the activity. We further confirmed that TA also strikingly inhibited the proliferation of several pancreatic cancer cell lines including CFPAC-1, AsPC-1, MIA PaCa-2, HPAC and SW1990 (Figure 2c). Unexpectedly, TA exhibited stronger proliferation suppressive activity against PANC-1 cells than other pancreatic cancer cell lines. Based on these results, the PANC-1 cells were selected for subsequent experiments. The effect of TA on the growth of PANC-1 cells was evaluated using the colony formation assay. As shown in Figure 2d, TA significantly inhibited the colony formation of PANC-1 cells compared with the control in a concentration-dependent manner. 
Additionally, the cell proliferation inhibitory activity of TA was better than that of BP-1-102, a specific STAT3 inhibitor, in pancreatic cancer (Figure S2a). In addition, we also examined whether TA restrained the proliferation of other cancer cells and normal cell lines. Interestingly, TA also strongly inhibited the proliferation of prostate and breast cancer cells rather than normal cell lines (Figure S2b). TA also significantly inhibited the colony formation of other pancreatic cancer cells compared with their control counterparts in a concentration-dependent way (Figure S3). These results indicated that TA exerted a strong inhibitory effect on the proliferation and growth of pancreatic cancer cells by inhibiting the STAT3 signaling pathway.

\subsection{TA induces cell-cycle arrest and suppresses the migration and invasion of PANC-1 cells}

To evaluate the influence of TA on the cell-cycle progression, the cell cycle distribution of PANC-1 cells was measured using the flow cytometric assay. Compared with the control group, the percentage of cells in S phase was apparently increased in the TA-treated groups (Figure 3a), which was probably associated with the downregulation of cell cycle regulation proteins (e.g. Cyclin D1 and c-Myc) by TA. To evaluate the effect of TA on the apoptosis of PANC-1 cells, the apoptosis rates were analyzed using the Annexin V-FITC/PI double staining assay. As shown in Figure 3b, the percentage of apoptotic cells was not significantly increased in the TA-treated groups compared with the control group. Although the expression of apoptosis-related proteins Survivin and Bcl2, was downregulated by TA, TA might mainly arrest cell cycle instead of inducing apoptosis to inhibit the proliferation and growth of PANC-1 cells through blocking the STAT3 pathway.

To investigate the effect of TA on the mobility of PANC-1 cells, the transwell migration assay was performed. Compared with the control group, the migration of PANC-1 cells in the TA-treated groups was effectively suppressed in a concentration-dependent manner (Figure 3c). Next, the Matrigel invasion assay was performed to evaluate the effect of TA on the invasion of PANC-1 cells. As shown in Figure 3d, the invasive ability of PANC-1 cells in the TA-treated groups was dramatically reduced in a dose-dependent manner compared with the control group. Thus, TA suppressed the migration and invasion capacities of PANC-1 cells by inhibiting the STAT3 signaling pathway. Furthermore, we examined whether TA inhibited the migration and invasion of other pancreatic cancer cells. As expected, TA also observably inhibited the migration and invasion of other pancreatic cancer cells in a dose-dependent manner (Figures S4 and S5). In brief, all of these data indicated that TA inhibited the proliferation, growth, migration and invasion of pancreatic cancer cells by inhibiting the STAT3 pathway.

\subsection{TA inhibits pancreatic tumor growth in vivo}

Since we observed the inhibition of proliferation and growth of pancreatic cancer cells after treatment with TA, we next sought to identify whether it would apparently repress pancreatic cancer xenograft tumor growth. We tested this hypothesis using a subcutaneous xenograft model of PANC-1 cells in nude mice. The tumorbearing nude mice were randomly divided into five groups: negative control group, positive control group, low-dose group, medium-dose group, and high-dose group. Gemcitabine hydrochloride, approved by the US Food and Drug Administration (FDA) as a first-line agent for the treatment of pancreatic cancer, was used as a positive control. The dosages of TA for the animal experiment were set at $10 \mathrm{mg} / \mathrm{kg} / \mathrm{d}, 20 \mathrm{mg} / \mathrm{kg} / \mathrm{d}$, and $40 \mathrm{mg} / \mathrm{kg} / \mathrm{d}$ based on our experience and pharmacological knowledge. As expected, intraperitoneal injection of TA dose-dependently caused significant inhibition of pancreatic tumor growth in the xenograft model. The inhibition of pancreatic tumor growth in the medium-dose group was as same as the positive control group (Figure 4a). As exhibited in Figure 4b,c, the tumor volume and tumor weight were significantly reduced in the TA-treated groups compared with the negative control group. H\&E staining of tumor tissue sections showed distinct tumor cell necrosis and immunohistological analysis indicated prominent reduction in the expression of proliferating cell nuclear antigen (PCNA) and Ki-67 within the tumor tissues of the TA-treated groups compared with tissues from the negative control group (Figure 4d). Importantly, there was no significant difference in body weight of mice among each group, indicating that TA had no apparent toxicity (Figure 4e). Furthermore, the pathological changes in the main organs of mice were also inspected by H\&E staining, and there was no significant toxicity at the effective dose (Figure 4f). In a word, TA markedly retarded tumor growth in vivo by inhibiting the proliferative capacity of PANC-1 cells, and had no obvious toxicity and side effects. 


\subsection{TA inhibits the STAT3 pathway in vivo}

We previously showed that TA inhibited the proliferation and growth of PANC-1 cells by down-regulating the expression levels of p-STAT3 (Tyr705) and STAT3-regulated proteins. To examine whether TA also suppressed the biological functions of STAT3 in vivo, we first evaluated the expression levels of total STAT3, p-STAT3 (Tyr705), and STAT3-regulated proteins in the tumor tissues. As shown in Figure 5a, TA evidently down-regulated the expression of p-STAT3 (Tyr705) and STAT3 downstream target proteins such as c-Myc, Survivin, and ICAM-1 in the high-dose group $(40 \mathrm{mg} / \mathrm{kg} / \mathrm{d})$, while had no remarkable effect on the protein expression of total STAT3, Cyclin D1, and Bcl-2. Furthermore, immunohistological analysis of tumor tissue sections also indicated that the expression of p-STAT3 (Tyr705), c-Myc, Survivin, MMP-9, and ICAM-1 was markedly reduced in the TA-treated groups compared with the negative control group (Figure $5 \mathrm{~b})$.

To further validate whether TA inhibited STAT3 signaling pathwayin vivo , the protein expression of pSTAT3 (Tyr705) was determined by the tissue immunofluorescence staining. TA significantly decreased the expression of p-STAT3 (Tyr705) protein in the tumor tissues (Figure S8a). In addition, the mRNA expression levels of STAT3 target genes were measured by the qRT-PCR to examine whether TA had an impact on the expression of STAT3 downstream genes in the tumor tissues. TA dramatically decreased endogenous BCL2 , MYC ,VEGF , CASP3 , MMP9 , ICAM1, and IL6mRNA expression, and upregulated CCND1 mRNA expression but had no impact on $M M P 2$ mRNA expression in the high-dose group $(40 \mathrm{mg} / \mathrm{kg} / \mathrm{d}$ ) (Figure S8b). In summary, our data demonstrated that TA substantially suppressed tumor growth via inhibiting STAT3 signaling pathway in vivo .

\subsection{Evaluation of TA pharmacokinetics in rats}

Bioactive compounds with excellent pharmacokinetic properties greatly increase the possibility of becoming new drugs. Since TA exhibited potent efficacy against pancreatic cancer both in vitro andin vivo, we also evaluated the pharmacokinetic properties of TA in Sprague-Dawley rats. As shown in Figure 6a, our studies indicated that the pharmacokinetic profiles of TA in rats after intravenous (i.v.) administration exhibited linear pharmacokinetics. However, we found that TA was slowly absorbed after intraperitoneal administration, which might be due to the poor water solubility of TA. After the sacrifice of the animals, tissue samples were collected to measure the tissue distribution levels of TA. Importantly, we found that TA showed good pancreatic tissue specificity (Figure 6b), which was beneficial to the treatment of pancreatic cancer. Moreover, pharmacokinetic parameters of TA after intravenous injection in rats were within the acceptance range (Figure 6c), suggesting that TA could be a potential drug candidate in pancreatic cancer.

\section{DISCUSSION}

Currently, the therapeutic options for patients with pancreatic cancer include surgical resection, chemotherapy, radiotherapy, and palliative care (Moore \& Donahue, 2019). Most patients with pancreatic cancer have no symptoms until the disease reaches the late stage at which tissue invasion and distant metastasis have been occurring. Chemotherapy is the predominant treatment for patients with metastatic pancreatic cancer (Neoptolemos et al., 2018). In the past thirty years, the standard drugs for treating pancreatic cancer were 5-fluorouracil (5-FU) and gemcitabine (Stan, Singh, \& Brand, 2010). In 1996, owing to the longer median survival time and higher quality of life, gemcitabine substituted 5-FU for the standard first-line chemotherapy agent (Ying, Zhu, \& Liu, 2012). Unfortunately, most of patients with advanced or metastatic pancreatic carcinoma could not gain benefits from gemcitabine monotherapy (Akinleye, Iragavarapu, Furqan, Cang, \& Liu, 2015). Furthermore, gemcitabine-based chemotherapy is associated with serious side effects and high resistance to the treatment (Adamska et al., 2018). Therefore, there is an unmet clinical need for effective chemotherapy for managing patients with pancreatic cancer. In this work, we identified a natural compound TA that strikingly inhibited pancreatic tumor growth both in vitro and in vivo, and significantly suppressed pancreatic cancer cell invasion and migration. In a subcutaneous tumor growth xenograft model of PANC-1 cells, we found that gemcitabine hydrochloride $(80 \mathrm{mg} / \mathrm{kg} / 3 \mathrm{~d})$ was as effective as TA $(20 \mathrm{mg} / \mathrm{kg} / \mathrm{d})$, which could remarkably inhibit the growth of pancreatic tumor and had no obvious toxicity. 
To further investigate the gene expression profiling of human pancreatic cancer cells under TA treatment, we performed RNA-seq after treating PANC-1 cells with or without TA. To validate the results of RNA-seq, significantly differentially expressed genes were selected for qRT-PCR verification. Indeed, as shown in Figure S7, the expression patterns of the tested genes were in accordance with the RNA-seq data, including 4 downregulated and 6 up-regulated genes. Moreover, we identified 2320 significantly differentially expressed genes, including 894 up-regulated genes and 1426 down-regulated genes (Figure S6a,b). Gene Ontology analysis demonstrated that these genes were primarily involved in basic processes (cell communication, biological regulation, signal transduction, etc.), suggesting that TA exhibited potent anticancer activity in pancreatic cancer by inhibiting critical processes (Figure S6c). KEGG pathway analysis suggested that these genes were mainly participated in cancer-related signaling pathways (metabolic pathways, pathways in cancer, JAK-STAT signaling pathway, etc.), supporting the potential therapeutic use of TA in pancreatic cancer (Figure S6d).

Under normal physiological conditions, STAT3 activation is rapid and lasts for only a few hours during signal transduction. Once activated by various upstream kinases, STAT3 protein is phosphorylated at the conserved tyrosine residue (Tyr705), leading to dimerization and nuclear translocation of p-STAT3, which subsequently binds to specific DNA sequences and induces transcription of STAT3 downstream target genes involved in a variety of biological processes (Avalle \& Poli, 2018; Swiatek-Machado \& Kaminska, 2020), such as cell cycle regulation (Cyclin D1 and c-Myc), evasion of apoptosis (Bcl-2 and Survivin), invasion and migration (MMP-9 and ICAM-1), and angiogenesis (VEGF and IL-8). Therefore, aberrantly activated STAT3 has been closely associated with the initiation, promotion and progression of various illnesses, especially cancer (Yu \& Jove, 2004). Recently, more and more studies have shown that STAT3 and its mediated signal transduction play a prominent role in the occurrence, development, invasion, and metastasis of pancreatic cancer (Huang \& Xie, 2012), and are also involved in the drug resistance of pancreatic cancer (Venkatasubbarao et al., 2013). In our research, we found that TA apparently reduced the transcriptional activity of STAT3 in a concentration-dependent manner. Furthermore, TA effectively inhibited the phosphorylation of tyrosine at the 705 site of STAT3 and the expression of downstream functional genes (e.g. Cyclin D1, c-Myc, Bcl-2, Survivin, and ICAM-1) at the protein levels, resulting in the growth inhibitory effects of TA on pancreatic cancer both in vitro and in vivo .

In fact, due to the pivotal role of STAT3 in tumor initiation and progression, a campaign in drug discovery has been launched to identify small molecules that interfere with the activation and function of STAT3 (Debnath, Xu, \& Neamati, 2012). Until now, a great quantity of STAT3 small-molecule inhibitors have been discovered and developed for clinical utility in treatment and prevention of malignant tumors (Yang et al., 2018). For instance, BP-1-102 is an orally bioavailable, highly potent and specific STAT3 inhibitor that has a very good clinical application prospect for cancer treatment (Zhang et al., 2012). Although several STAT3 inhibitors have entered the early clinical stage, there currently are no FDA-approved small molecule inhibitors (Beebe, Liu, \& Zhang, 2018). Hence it is of great significance to find potential and effective STAT3 inhibitors. First of all, we predicted the theoretical binding mode between the natural compound TA and STAT3 protein by molecular docking, and subsequently demonstrated that TA was able to bind to STAT3 (127-722 AA) protein by SPR assay. More importantly, we found that the cell proliferation activity of TA was much better than that of BP-1-102 in pancreatic cancer.

Although we reported the potential target protein of TA for the first time, its affinity with STAT3 protein needs to be further improved, and the binding site with STAT3 protein also needs to be further studied. In addition, the water solubility of TA should be further improved, and the molecular mechanisms of TA against pancreatic cancer should also be further clarified.

In summary, we screened and characterized the natural compound TA, a potential STAT3 pathway inhibitor that restrained the growth of pancreatic cancer both in vitro and in vivo. TA was efficacious in suppressing the migration and invasion of pancreatic cancer cells via inhibiting the STAT3 pathway. More than anything, we first revealed the underlying molecular mechanisms of TA against pancreatic cancer (Figure 7). TA can be a new potential candidate compound for treating pancreatic cancer, and it is valuable to be further 
investigated.

\section{REFERENCES}

Kleeff, J., Korc, M., Apte, M., La Vecchia, C., Johnson, C. D., Biankin, A. V., .. Neoptolemos, J. P. (2016). Pancreatic cancer.Nature Reviews Disease Primers , 2 , 16022.https://doi.org/10.1038/nrdp.2016.22

Aier, I., Semwal, R., Sharma, A., \& Varadwaj, P. K. (2019). A systematic assessment of statistics, risk factors, and underlying features involved in pancreatic cancer. Cancer Epidemiology ,58 , 104110.https://doi.org/10.1016/j.canep.2018.12.001

Bray, F., Ferlay, J., Soerjomataram, I., Siegel, R. L., Torre, L. A., \& Jemal, A. (2018). Global cancer statistics 2018: GLOBOCAN estimates of incidence and mortality worldwide for 36 cancers in 185 countries. $C A-A$ Cancer Journal for Clinicians , 68 , 394-424.https://doi.org/10.3322/caac.21492

Siegel, R. L., Miller, K. D., \& Jemal, A. (2020). Cancer statistics, 2020. CA-A Cancer Journal for Clinicians , 70 , 7-30.https://doi.org/10.3322/caac.21590

Rahib, L., Smith, B. D., Aizenberg, R., Rosenzweig, A. B., Fleshman, J. M., \& Matrisian, L. M. (2014). Projecting cancer incidence and deaths to 2030: the unexpected burden of thyroid, liver, and pancreas cancers in the United States. Cancer Research, 74, 2913-21.https://doi.org/10.1158/0008-5472.CAN-14-0155

Roth, M. T., Cardin, D. B., \& Berlin, J. D. (2020). Recent advances in the treatment of pancreatic cancer. F1000Research, 9 , F1000 Faculty Rev-131.https://doi.org/10.12688/f1000 research.21981.1

Kamisawa, T., Wood, L. D., Itoi, T., \& Takaori, K. (2016). Pancreatic cancer. Lancet , 388 , $73-$ 85.https://doi.org/10.1016/S0140-6736(16)00141-0

Binenbaum, Y., Na'ara, S., \& Gil, Z. (2015). Gemcitabine resistance in pancreatic ductal adenocarcinoma. Drug Resistance Updates ,23, 55-68.https://doi.org/10.1016/j.drup.2015.10.002

Dutta, P., Sabri, N., Li, J., \& Li, W. X. (2015). Role of STAT3 in lung cancer. JAKSTAT , 3 , e999503.https://doi.org/10.1080/21623996.2014.999503

Banerjee, K., \& Resat, H. (2016). Constitutive activation of STAT3 in breast cancer cells: A review. International Journal of Cancer ,138, 2570-8.https://doi.org/10.1002/ijc.29923

Ouédraogo, Z. G., Biau, J., Kemeny, J. L., Morel, L., Verrelle, P., \& Chautard, E. (2017). Role of STAT3 in Genesis and Progression of Human Malignant Gliomas. Molecular Neurobiology , 54 , 57805797.https://doi.org/10.1007/s12035-016-0103-0

Liang, R., Chen, X., Chen, L., Wan, F., Chen, K., Sun, Y., \& Zhu, X. (2020). STAT3 signaling in ovarian cancer: a potential therapeutic target. Journal of Cancer , 11 , 837-848.https://doi.org/10.7150/jca.35011

Carpenter, R. L., \& Lo, H. W. (2014). STAT3 Target Genes Relevant to Human Cancers. Cancers (Basel), 6, 897-925.https://doi.org/10.3390/cancers6020897

Kamran, M. Z., Patil, P., \& Gude, R. P. (2013). Role of STAT3 in cancer metastasis and translational advances. Biomed Research International , 2013 , 421821.https://doi.org/10.1155/2013/421821

Wendt, M. K., Balanis, N., Carlin, C. R., \& Schiemann, W. P. (2014). STAT3 and epithelial- mesenchymal transitions in carcinomas.JAKSTAT , 3 , e28975.https://doi.org/10.4161/jkst.28975

Gao, P., Niu, N., Wei, T., Tozawa, H., Chen, X., Zhang, C., .. Liu, J. (2017). The roles of signal transducer and activator of transcription factor 3 in tumor angiogenesis. Oncotarget ,8 , 6913969161.https://doi.org/10.18632/oncotarget.19932

Demaria, M., Giorgi, C., Lebiedzinska, M., Esposito, G., D’Angeli, L., Bartoli, A., ... Poli, V. (2010). A STAT3-mediated metabolic switch is involved in tumour transformation and STAT3 addiction. Aging (Albany NY) , 2 , 823-42.https://doi.org/10.18632/aging.100232 
Galoczova, M., Coates, P., \& Vojtesek, B. (2018). STAT3, stem cells, cancer stem cells and p63. Cellular $\mathcal{6}$ Molecular Biology Letters ,23, 12.https://doi.org/10.1186/s11658-018-0078-0

Wang, T., Niu, G., Kortylewski, M., Burdelya, L., Shain, K., Zhang, S., .. Yu, H. (2004). Regulation of the innate and adaptive immune responses by Stat-3 signaling in tumor cells. Nature Medicine ,10 , 48-54.https://doi.org/10.1038/nm976

Fan, Y., Mao, R., \& Yang, J. (2013). NF- $\varkappa$ B and STAT3 signaling pathways collaboratively link inflammation to cancer. Protein \& Cell ,4 , 176-85.https://doi.org/10.1007/s13238-013-2084-3

Yu, H., Lee, H., Herrmann, A., Buettner, R., \& Jove, R. (2014). Revisiting STAT3 signalling in cancer: new and unexpected biological functions. Nature Reviews Cancer , 14 , 736-746.https://doi.org/10.1038/nrc3818

Yu, H., Pardoll, D., \& Jove, R. (2009). STATs in cancer inflammation and immunity: a leading role for STAT3. Nature Reviews Cancer ,9 , 798-809.https://doi.org/10.1038/nrc2734

Wang, Y., Shen, Y., Wang, S., Shen, Q., \& Zhou, X. (2018). The role of STAT3 in leading the crosstalk between human cancers and the immune system. Cancer Letters , 415 , 117128.https://doi.org/10.1016/j.canlet.2017.12.003

Yu, H., Kortylewski, M., \& Pardoll, D. (2007). Crosstalk between cancer and immune cells: role of STAT3 in the tumour microenvironment.Nature Reviews Immunology , 7 , 41-51.https://doi.org/10.1038/nri1995

Lee, H. J., Zhuang, G., Cao, Y., Du, P., Kim, H. J., \& Settleman, J. (2014). Drug resistance via feedback activation of Stat3 in oncogene-addicted cancer cells. Cancer Cell , 26 , 20721.https://doi.org/10.1016/j.ccr.2014.05.019

Zhao, C., Li, H., Lin, H. J., Yang, S., Lin, J., \& Liang, G. (2016). Feedback Activation of STAT3 as a Cancer Drug-Resistance Mechanism.Trends in Pharmacological Sciences , 37 , 4761.https://doi.org/10.1016/j.tips.2015.10.001

Johnson, D. E., O'Keefe, R. A., \& Grandis, J. R. (2018). Targeting the IL-6/JAK/STAT3 signalling axis in cancer. Nature Reviews Clinical Oncology , 15 , 234-248.https://doi.org/10.1038/nrclinonc.2018.8

Furtek, S. L., Backos, D. S., Matheson, C. J., \& Reigan, P. (2016). Strategies and Approaches of Targeting STAT3 for Cancer Treatment.ACS Chemical Biology , 11 , 30818.https://doi.org/10.1021/acschembio.5b00945

Scholz, A., Heinze, S., Detjen, K. M., Peters, M., Welzel, M., Hauff, P., .. Rosewicz, S. (2003). Activated signal transducer and activator of transcription 3 (STAT3) supports the malignant phenotype of human pancreatic cancer. Gastroenterology , 125 , 891-905.https://doi.org/10.1016/s0016-5085(03)01064-3

Fofaria, N. M., \& Srivastava, S. K. (2015). STAT3 induces anoikis resistance, promotes cell invasion and metastatic potential in pancreatic cancer cells. Carcinogenesis , 36 , 14250.https://doi.org/10.1093/carcin/bgu233

He, W., Wu, J., Shi, J., Huo, Y. M., Dai, W., Geng, J., .. Xue, J. (2018). IL22RA1/STAT3 Signaling Promotes Stemness and Tumorigenicity in Pancreatic Cancer. Cancer Research , 78 , 32933305.https://doi.org/10.1158/0008-5472.CAN-17-3131

Wei, D., Le, X., Zheng, L., Wang, L., Frey, J. A., Gao, A. C., ... Xie, K. (2003). Stat3 activation regulates the expression of vascular endothelial growth factor and human pancreatic cancer angiogenesis and metastasis. Oncogene, 22, 319-29.https://doi.org/10.1038/sj.onc.1206122

Li, N., Grivennikov, S. I., \& Karin, M. (2011). The unholy trinity: inflammation, cytokines, and STAT3 shape the cancer microenvironment.Cancer Cell , 19 , 429-31.https://doi.org/10.1016/j.ccr.2011.03.018

D'Amico, S., Shi, J., Martin, B. L., Crawford. H. C., Petrenko, O., \& Reich, N. C. (2018). STAT3 is a master regulator of epithelial identity and KRAS-driven tumorigenesis. Genes 85 Development, 32 , 
Tang, D., Liu, L. L., He, Q. R., Yan, W., Li, D., \& Gao, J. M. (2018). Ansamycins with Antiproliferative and Antineuroinflammatory Activity from Moss-Soil-Derived Streptomyces cacaoi subsp. asoensis H2S5. Journal of Natural Products , 81 , 1984-1991.https://doi.org/10.1021/acs.jnatprod.8b00203

Liu, L. L., Chen, Z. F., Liu, Y., Tang, D., Gao, H. H., Zhang, Q., \& Gao, J. M. (2020). Molecular networking-based for the target discovery of potent antiproliferative polycyclic macrolactam ansamycins from Streptomyces cacaoi subsp. Asoensis. Organic Chemistry Frontiers ,https://doi.org/10.1039/D0QO00557F

Alexander, S. P. H., Roberts, R. E., Broughton, B. R. S., Sobey, C. G., George, C. H., Stanford, S. C., ... Ahluwalia, A. (2018). Goals and practicalities of immunoblotting and immunohistochemistry: A guide for submission to the British Journal of Pharmacology. British Journal of Pharmacology , 175 , 407411.https://doi.org/10.1111/bph.14112

Kilkenny, C., Browne, W., Cuthill, I. C., Emerson, M., \& Altman, D. G. (2010). Animal research: Reporting in vivo experiments: The ARRIVE guidelines. British Journal of Pharmacology , 160 , 15771579.https://doi.org/10.1111/j.1476-5381.2010.00872.x

McGrath, J. C., \& Lilley, E. (2015). Implementing guidelines on reporting research using animals (ARRIVE etc.): new requirements for publication in BJP. British Journal of Pharmacology , 172 , 31893193.https://doi.org/10.1111/bph.12955

Curtis, M. J., Alexander, S., Cirino, G., Docherty, J. R., George, C. H., Giembycz, M. A., ... Ahluwalia, A. (2018). Experimental design and analysis and their reporting II: updated and simplified guidance for authors and peer reviewers. British Journal of Pharmacology ,175, 987-993.https://doi.org/10.1111/bph.14153

Bharadwaj, U., Eckols, T. K., Xu, X., Kasembeli, M. M., Chen, Y., Adachi, M., ... Tweardy, D. J. (2016). Small-molecule inhibition of STAT3 in radioresistant head and neck squamous cell carcinoma. Oncotarget , 7, 26307-30.https://doi.org/10.18632/oncotarget.8368

Zhang, X., Yue, P., Page, B. D., Li, T., Zhao, W., Namanja, A. T., ... Turkson, J. (2012). Orally bioavailable small-molecule inhibitor of transcription factor Stat3 regresses human breast and lung cancer xenografts. Proceedings of the National Academy of Sciences of the United States of America , 109, 96238.https://doi.org/10.1073/pnas.1121606109

Moore, A., \& Donahue, T. (2019). Pancreatic Cancer. JAMA-Journal of the American Medical Association , 322, 1426.https://doi.org/10.1001/jama.2019.14699

Neoptolemos, J. P., Kleeff, J., Michl, P., Costello, E., Greenhalf, W., \& Palmer, D. H. (2018). Therapeutic developments in pancreatic cancer: current and future perspectives. Nature Reviews Gastroenterology $\mathscr{B}$ Hepatology , 15 , 333-348.https://doi.org/10.1038/s41575-018-0005-x

Stan, S. D., Singh, S. V., \& Brand, R. E. (2010). Chemoprevention strategies for pancreatic cancer. Nature Reviews Gastroenterology 83 Hepatology, 7 , 347-356.https://doi.org/10.1038/nrgastro.2010.61

Ying, J. E., Zhu, L. M., \& Liu, B. X. (2012). Developments in metastatic pancreatic cancer: is gemcitabine still the standard? World Journal of Gastroenterology , 18, 736-45.https://doi.org/10.3748/wjg.v18.i8. 736

Akinleye, A., Iragavarapu, C., Furqan, M., Cang, S., \& Liu, D. (2015). Novel agents for advanced pancreatic cancer. Oncotarget ,6 ,39521-39537.https://doi.org/10.18632/oncotarget.3999

Adamska, A., Elaskalani, O., Emmanouilidi, A., Kim, M., Razak, N. B. A., Metharom, P., \& Falasca, M. (2018). Molecular and cellular mechanisms of chemoresistance in pancreatic cancer. Advances in Biological Regulation , 68 , 77-87.https://doi.org/10.1016/j.jbior.2017.11.007

Avalle, L., \& Poli, V. (2018). Nucleus, Mitochondrion, or Reticulum? STAT3 à La Carte. International Journal of Molecular Sciences ,19 , 2820.https://doi.org/10.3390/ijms19092820 
Swiatek-Machado, K., \& Kaminska, B. (2020). STAT Signaling in Glioma Cells. Advances in Experimental Medicine and Biology ,1202, 203-222.https://doi.org/10.1007/978-3-030-30651-9-10

Yu, H., \& Jove, R. (2004). The STATs of cancer-new molecular targets come of age. Nature Reviews Cancer , 4, 97-105.https://doi.org/10.1038/nrc1275

Huang, C., \& Xie, K. (2012). Crosstalk of Sp1 and Stat3 signaling in pancreatic cancer pathogenesis. Cytokine 83 Growth Factor Reviews, 23, 25-35.https://doi.org/10.1016/j.cytogfr.2012.01.003

Venkatasubbarao, K., Peterson, L., Zhao, S., Hill, P., Cao, L., Zhou, Q., .. Freeman, J. W. (2013). Inhibiting signal transducer and activator of transcription-3 increases response to gemcitabine and delays progression of pancreatic cancer. Molecular Cancer ,12, 104.https://doi.org/10.1186/1476-4598-12-104

Debnath, B., Xu, S., \& Neamati, N. (2012). Small molecule inhibitors of signal transducer and activator of transcription 3 (Stat3) protein.Journal of Medicinal Chemistry , 55 , 66456668.https://doi.org/10.1021/jm30020\%s

Yang, L., Lin, S., Xu, L., Lin, J., Zhao, C., \& Huang, X. (2018). Novel activators and small-molecule inhibitors of STAT3 in cancer.Cytokine $\&$ Growth Factor Reviews , 49 , 1022.https://doi.org/10.1016/j.cytogfr.2019.10.005

Beebe, J. D., Liu, J. Y., \& Zhang, J. T. (2018). Two decades of research in discovery of anticancer drugs targeting STAT3, how close are we? Pharmacology \& Therapeutics , 191 , 7491.https://doi.org/10.1016/j.pharmthera.2018.06.006

Figure legends:
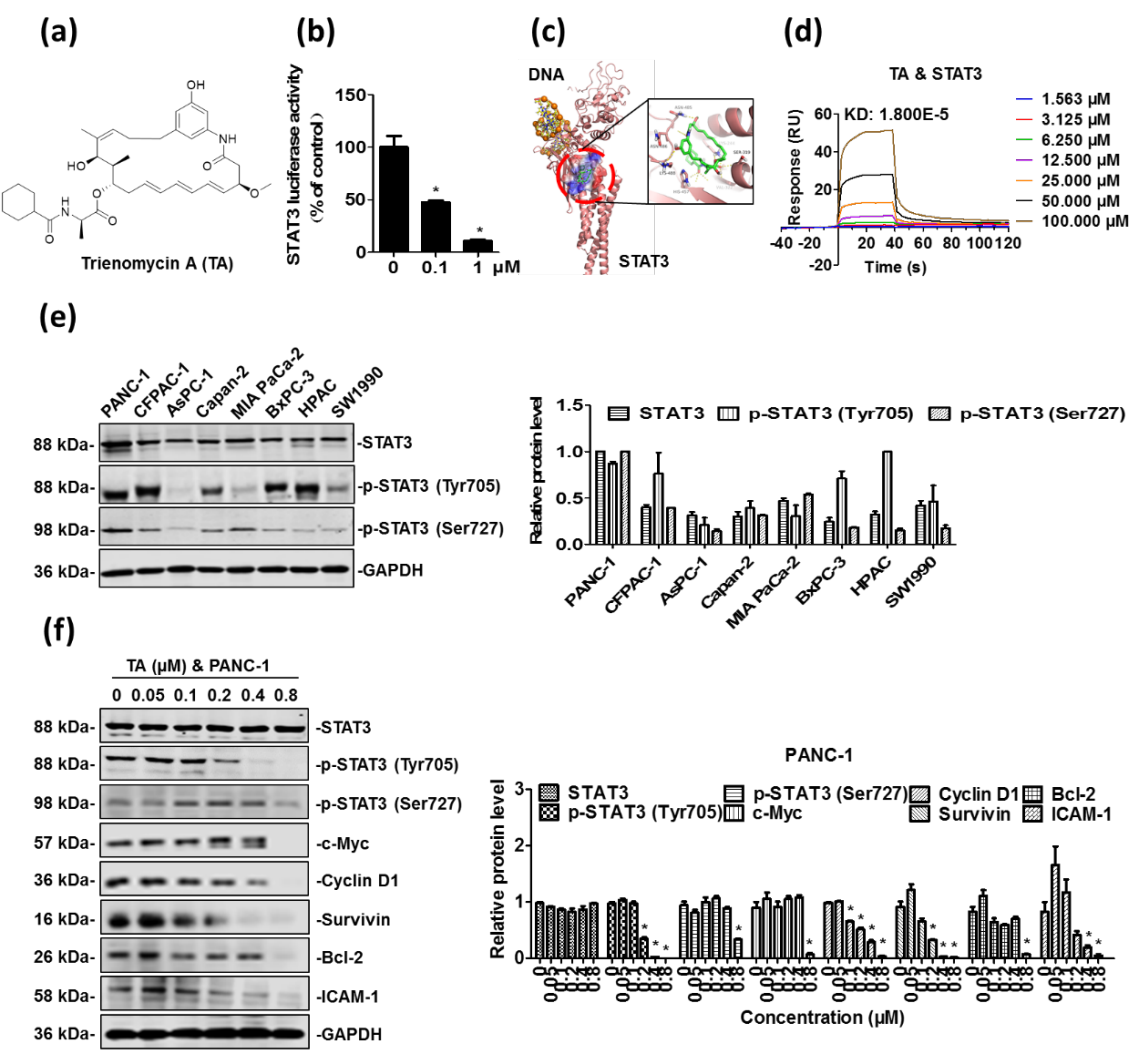

FIGURE 1 TA markedly suppresses the STAT3 signaling pathway. (a) Chemical structure of TA. (b) 
STAT3 luciferase assay in 293T cells. 293T cells with a transfected STAT3 luciferase reporter were stimulated with IL-6 $(20 \mathrm{ng} / \mathrm{mL})$ and different dose of TA $(0,0.1$, and $1 \mu \mathrm{M})$ for $12 \mathrm{~h}$. Normalized luminescence was plotted as STAT3 luciferase activity compared to control (\%). Data are expressed as means \pm SD $(n=5)$. ${ }^{*} P<0.05$. (c) Modeling of TA binding with STAT3 (PDB: 1BG1). Left: TA binding with the STAT3ß/DNA complex. Right: Ligand interaction diagram of the binding. The STAT3 structure was shown as ribbon representation and TA was shown as stick. (d) Interaction of TA with STAT3 in vitro . Surface plasmon resonance (SPR) assays of the immobilized STAT3 (127-722 AA) toward varied concentrations of TA (1.56$100 \mu \mathrm{M})$. (e) Left: Expression levels of STAT3 and p-STAT3 (Tyr705 and Ser727) in eight pancreatic cancer cell lines (PANC-1, CFPAC-1, AsPC-1, Capan-2, MIA PaCa-2, BxPC-3, HPAC and SW1990). GAPDH was used as a loading control. Right: Analysis of the results in (e). Protein levels were quantified by using Image J software. Data are expressed as means $\pm \mathrm{SD}(\mathrm{n}=5)$. (f) Left: Effect of TA on the STAT3, p-STAT3 (Tyr705 and Ser727), and STAT3-regulated proteins in PANC-1 cells. The cells were treated with several concentrations of TA $(0-0.8 \mu \mathrm{M})$ for $24 \mathrm{~h}$. Whole cell lysates were subjected to western blotting for STAT3, p-STAT3 (Tyr705 and Ser727), c-Myc, Bcl-2, Cyclin D1, Survivin and ICAM-1. GAPDH was used here as a loading control. Right: Analysis of the results in (f). Protein levels were quantified by using ImageJ software. Data are expressed as means $\pm \mathrm{SD}(\mathrm{n}=5) .{ }^{*} P<0.05$.

(a)

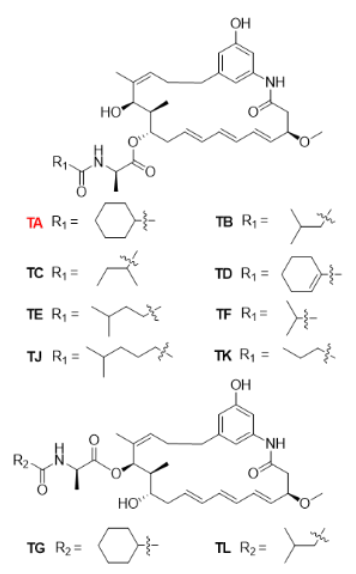

(c)

\begin{tabular}{|c|c|c|}
\hline Cell lines & \multicolumn{2}{|c|}{ TA } \\
\hline IC $50(\mu \mathrm{M})$ & $48 \mathrm{~h}$ & $72 \mathrm{~h}$ \\
\hline PANC-1 & $0.20 \pm 0.052$ & $0.070 \pm 0.014$ \\
\hline CFPAC-1 & $1.04 \pm 0.015$ & $0.33 \pm 0.040$ \\
\hline AsPC-1 & $2.12 \pm 0.54$ & $0.45 \pm 0.11$ \\
\hline Capan-2 & $1.85 \pm 1.05$ & $0.48 \pm 0.035$ \\
\hline MIA PaCa-2 & $0.54 \pm 0.11$ & $0.14 \pm 0.015$ \\
\hline BxPC-3 & $0.63 \pm 0.41$ & $0.11 \pm 0.010$ \\
\hline HPAC & $1.02 \pm 0.11$ & $0.39 \pm 0.070$ \\
\hline SW1990 & $2.42 \pm 0.075$ & $0.92 \pm 0.16$ \\
\hline
\end{tabular}

\section{(b)}

\begin{tabular}{|c|c|c|c|}
\hline $\begin{array}{c}\text { Compounds } \\
\text { IC50 }(\mu \mathrm{M})\end{array}$ & PANC-1 & Capan-2 & BxPC-3 \\
\hline TA & $0.20 \pm 0.052$ & $1.85 \pm 1.05$ & $0.63 \pm 0.41$ \\
\hline TB & $7.45 \pm 1.80$ & $9.91 \pm 0.09$ & $5.88 \pm 2.55$ \\
\hline TC & $3.98 \pm 0.34$ & $9.47 \pm 0.48$ & $2.10 \pm 0.93$ \\
\hline TD & $1.78 \pm 1.35$ & $3.72 \pm 3.04$ & $1.30 \pm 1.20$ \\
\hline TE & $2.17 \pm 0.47$ & $4.23 \pm 1.47$ & $1.16 \pm 0.80$ \\
\hline TF & $>10$ & $>10$ & $9.76 \pm 0.25$ \\
\hline TG & $>10$ & $>10$ & $>10$ \\
\hline TJ & $8.95 \pm 0.30$ & $>10$ & $7.16 \pm 1.22$ \\
\hline TK & $2.84 \pm 0.12$ & $6.38 \pm 2.33$ & $2.04 \pm 0.74$ \\
\hline TL & $>10$ & $>10$ & $>10$ \\
\hline
\end{tabular}

(d)

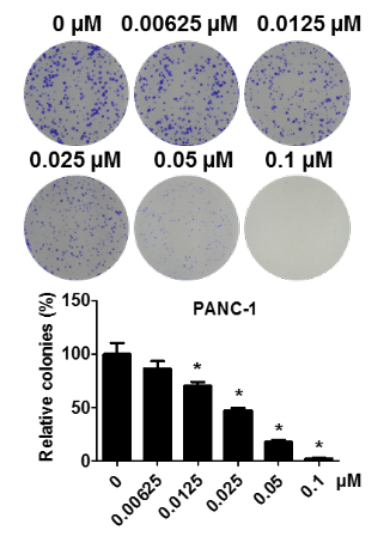

FIGURE 2 TA significantly inhibits the proliferation and growth of pancreatic cancer cells. (a) Chemical structures of Trienomycins A, B, C, D, E, F, G, J, K, and L. (b) Effect of trienomycins on the proliferation 
of pancreatic cancer cells. Cells were treated with different concentrations of trienomycins $(0-10 \mu \mathrm{M})$ for 48 h. Cell viability was measured with MTS assay. Data are shown as means $\pm \mathrm{SD}(\mathrm{n}=5)$. (c) Effect of TA on the proliferation of pancreatic cancer cells. Cells were treated with different concentrations of TA (0-10 $\mu \mathrm{M})$ for $48 \mathrm{~h}$ or $72 \mathrm{~h}$. Cell viability was measured with MTS assay. Data are shown as means $\pm \mathrm{SD}(\mathrm{n}=$ 5). (d) Up: Effect of TA on the colony formation of PANC-1 cells. Cells were seeded into six-well plates and treated with different concentrations of TA $(0-0.1 \mu \mathrm{M})$ for 7 days. Colonies were stained with crystal violet and photographed by using a digital camera. Down: Analysis of the results in (d). Colonies were counted by using ImageJ software. Data are shown as means $\pm \mathrm{SD}(\mathrm{n}=5) .{ }^{*} P<0.05$.

(a)

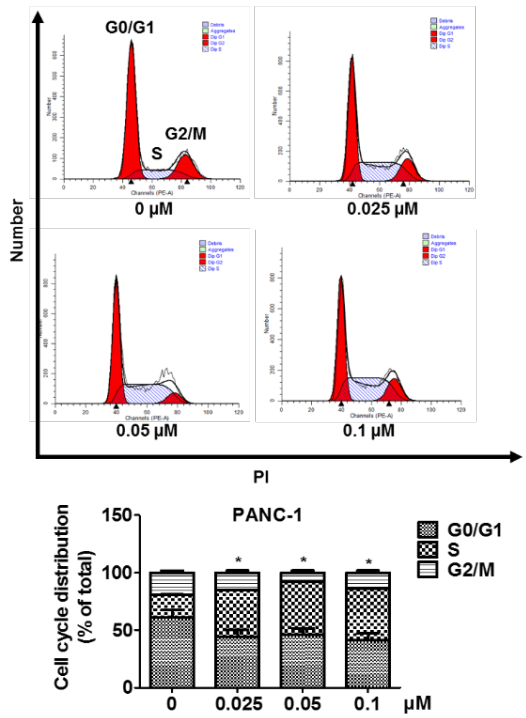

(b)

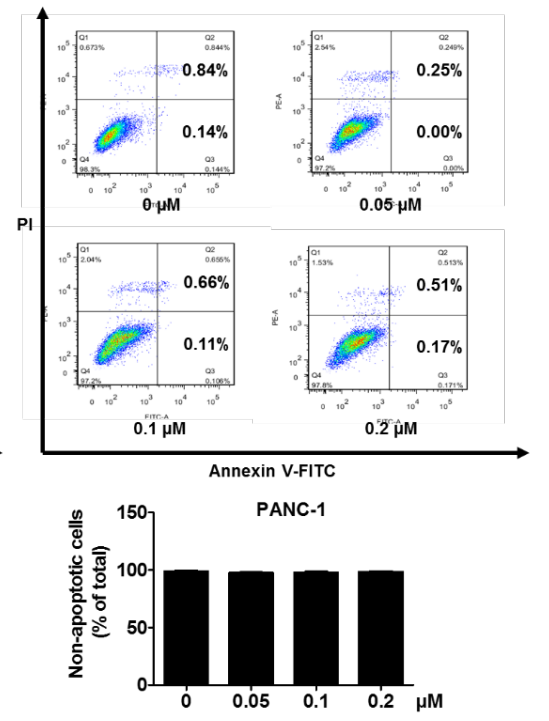

(c)

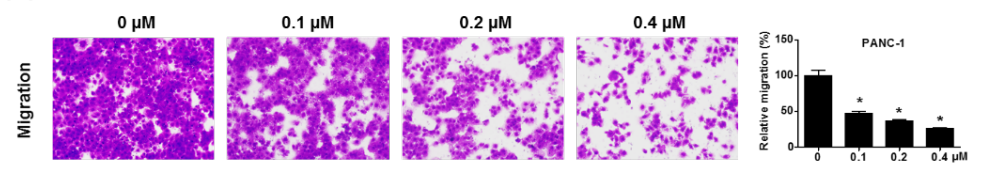

(d)

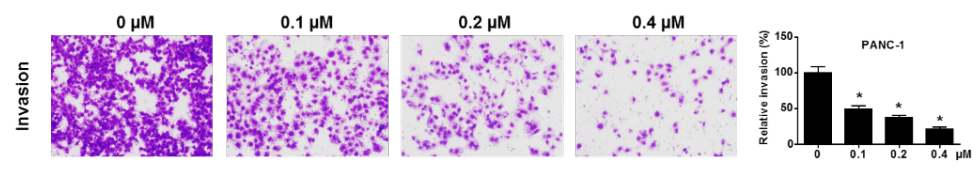

FIGURE 3 TA arrests cell cycle and inhibits the migration and invasion of PANC-1 cells. (a) Up: Effect of TA on the cell cycle of PANC-1 cells at the indicated concentrations. Cells were seeded in $6 \mathrm{~cm}$ dishes and incubated with the indicated concentrations of TA $(0-0.1 \mu \mathrm{M})$ for $24 \mathrm{~h}$. Cellular DNA was stained with PI, and flow cytometry analysis was performed to determine cell-cycle distribution. Down: Analysis of the results in (a). Data were analyzed by using ModFit LT software. Data are shown as means \pm SEM $(\mathrm{n}=$ 5). ${ }^{*} P<0.05$. (b) Up: Effect of TA on the apoptosis of PANC-1 cells at the indicated concentrations. Cells were cultured in six-well plates and treated with the indicated concentrations of TA $(0-0.2 \mu \mathrm{M})$ for $48 \mathrm{~h}$. Following the annexin V-FITC and PI staining procedure, flow cytometry analysis was performed to detect the apoptosis rate. Down: Analysis of the results in (b). Data were analyzed by using FlowJo software. Data are shown as means \pm SEM $(n=5)$. (c) Left: Effect of TA on the migration of PANC-1 cells. Cells were treated with various concentrations of TA $(0-0.4 \mu \mathrm{M})$ and seeded on the upper chambers. After an 
incubation of $48 \mathrm{~h}$, cells on the lower surface of the membrane were stained with $0.1 \%$ crystal violet. Scale bar, $50 \mu \mathrm{m}$. Right: Analysis of the results in (c). Migrated cells were counted by using ImageJ software. Data are expressed as means $\pm \mathrm{SD}(\mathrm{n}=5) .{ }^{*} P<0.05$. (d) Left: Effect of TA on the invasion of PANC-1 cells. Cells were treated with various concentrations of TA $(0-0.4 \mu \mathrm{M})$ and seeded on the upper chambers pre-coated with $50 \mu \mathrm{L}$ of Matrigel. After an incubation of $48 \mathrm{~h}$, cells on the lower surface of the membrane were stained with $0.1 \%$ crystal violet. Scale bar, $50 \mu \mathrm{m}$. Right: Analysis of the results in (d). Invasive cells were counted by using ImageJ software. Data are expressed as means $\pm \mathrm{SD}(\mathrm{n}=5)$. ${ }^{*} P<0.05$.

(a)

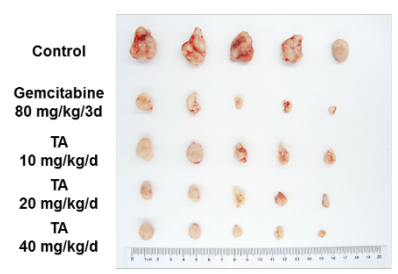

(d) (b)

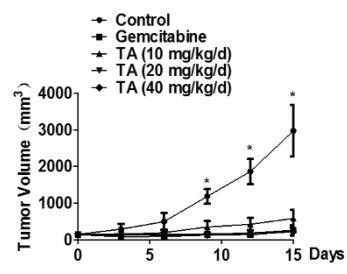

(c)

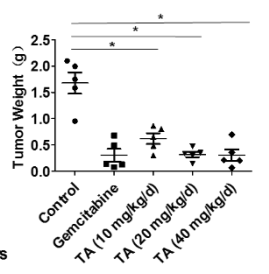

(e)
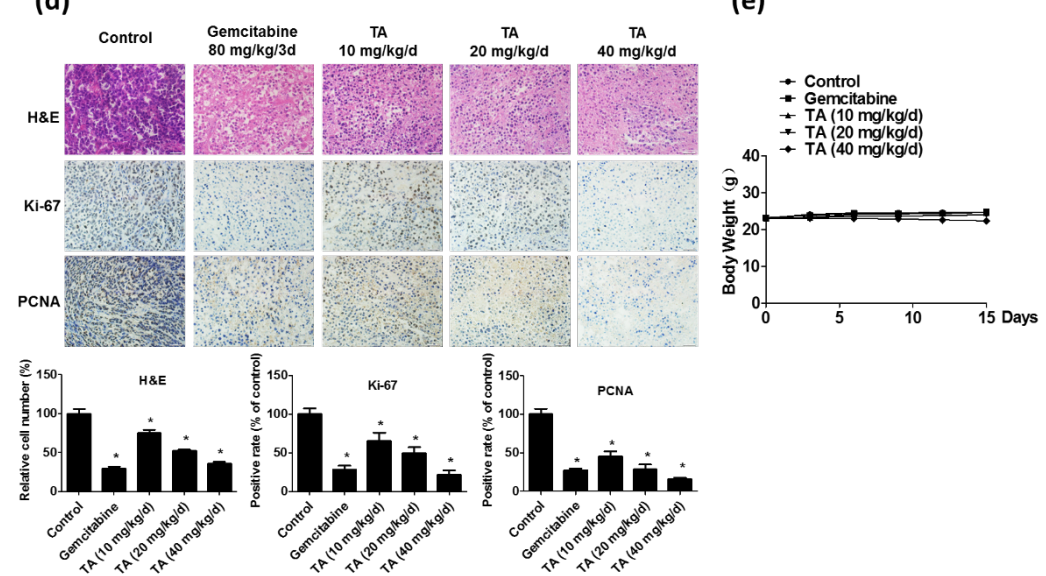

(f)

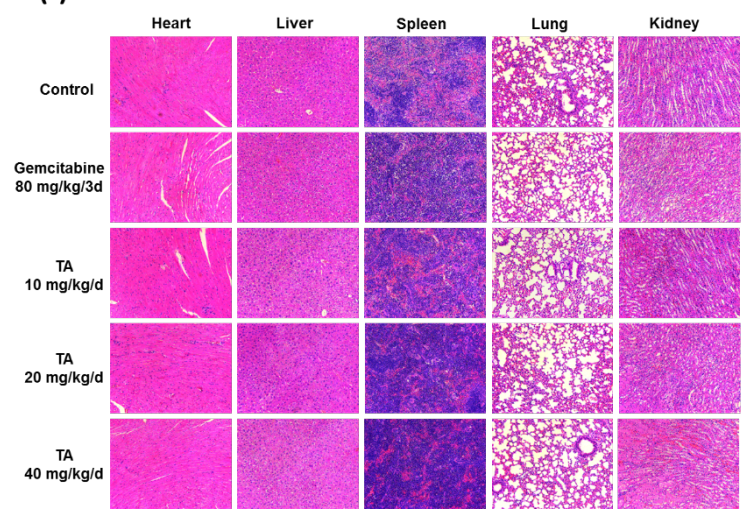

FIGURE 4 TA substantially suppresses pancreatic tumor growthin vivo . Male BALB/c nude mice were subcutaneously injected with PANC- 1 cells and intraperitoneally administered with 10,20 , and $40 \mathrm{mg} / \mathrm{kg} / \mathrm{d}$ of TA or vehicle control for 15 days. Gemcitabine hydrochloride $(80 \mathrm{mg} / \mathrm{kg} / 3 \mathrm{~d})$ was used as a positive drug. (a) Effect of TA on the pancreatic tumor growth. At the end of the experiment, the tumors were harvested. The picture was taken using a digital camera. (b) Tumor volume was measured once every 3 days. Data are expressed as means $\pm \mathrm{SD}(\mathrm{n}=5) .{ }^{*} P<0.05$. (c) Tumor weight was determined at the last day indicated. 
Data are expressed as means $\pm \mathrm{SD}(\mathrm{n}=5) .{ }^{*} P<0.05$. (d) Up: H\&E staining and immunohistochemical analysis of tumor tissues. Tumor tissues were fixed, dehydrated, and embedded into paraffin blocks. Tissue sections were cut from paraffin blocks, stained with hematoxylin and eosin (H\&E), or immunolabeled to detect the expression of Ki67 and PCNA. Scale bar, $50 \mu \mathrm{m}$. Down: Analysis of the results in (d). Positive cells were counted by using ImageJ software. Data are expressed as means $\pm \mathrm{SD}(\mathrm{n}=5)$. ${ }^{*} P<0.05$. (e) Body weight was measured once every 3 days. Data are expressed as means $\pm \mathrm{SD}(\mathrm{n}=5)$. (f) Heart, liver, spleen, lung and kidney of mice were fixed, dehydrated, and embedded into paraffin blocks. Tissue sections were cut from paraffin blocks and used for H\&E staining. Scale bar, $100 \mu \mathrm{m}$.

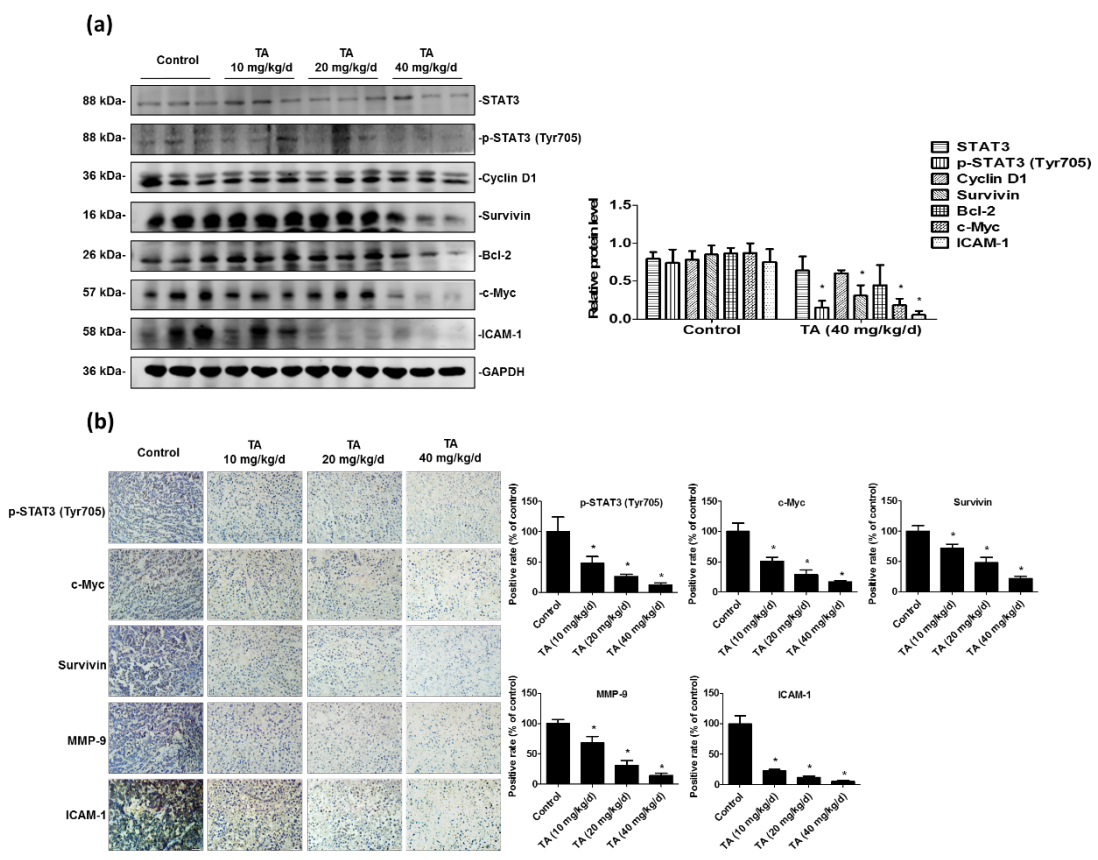

FIGURE 5 TA inhibits the STAT3 signaling pathway in vivo . (a) Left: STAT3-regulated protein expression in tumor tissues. The protein levels of STAT3, p-STAT3 (Tyr705), Survivin, Bcl-2, c-Myc, ICAM-1, and Cyclin D1 in the tumor tissues were assessed using western blotting. Right: Analysis of the results in (a). Protein levels were quantified by using ImageJ software. Data are shown as means \pm SD $(n=5)$. ${ }^{*} P<0.05$. (b) Left: Immunohistochemical analysis of tumor tissues. Tumor tissues were fixed, dehydrated, and embedded into paraffin blocks. Tissue sections were cut from paraffin blocks and immunolabeled to detect the expression of p-STAT3 (Tyr705), c-Myc, Survivin, MMP-9, and ICAM-1. Scale bar, $50 \mu \mathrm{m}$. Right: Analysis of the results in (b). Positive cells were counted by using ImageJ software. Data are shown as means $\pm \mathrm{SD}(\mathrm{n}=5) .{ }^{*} P<0.05$. 
(a)

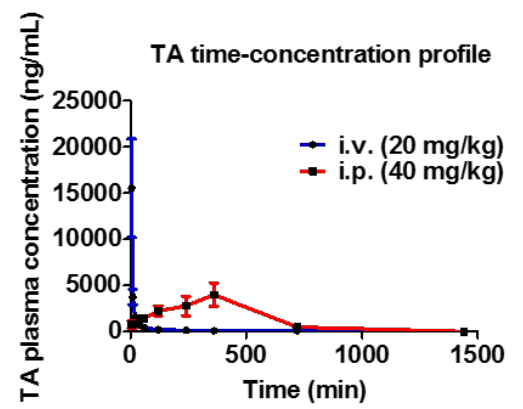

(b)

\begin{tabular}{|c|c|}
\hline Tissues & Concentrations $(\mathrm{ng} / \mathrm{mg})$ \\
\hline brain & $0.04 \pm 0.02$ \\
\hline kidney & $3.69 \pm 1.59$ \\
\hline liver & $7.28 \pm 6.13$ \\
\hline pancreas & $34.76 \pm 5.71$ \\
\hline
\end{tabular}

\section{(c)}

\begin{tabular}{c|c|c|}
$\begin{array}{c}\text { Pharmacokinetic } \\
\text { parameters of TA }\end{array}$ & Units & $\begin{array}{c}\text { i.v. }(20 \mathrm{mg} / \mathrm{kg}) \\
\mathrm{n}=6\end{array}$ \\
\hline $\mathrm{t}_{1 / 2}$ & $\mathrm{~min}$ & $467.90 \pm 96.87$ \\
\hline $\mathrm{t}_{\max }$ & $\mathrm{min}$ & $4.98 \pm 0.00$ \\
\hline $\mathrm{C}_{\max }$ & $\mathrm{ng} / \mathrm{mL}$ & $15548.02 \pm 5829.10$ \\
\hline $\mathrm{AUC}_{0-24}$ & $\mathrm{~min} \cdot \mathrm{ng} / \mathrm{mL}$ & $453371.31 \pm 213938.78$ \\
\hline $\mathrm{AUC}_{0-\infty}$ & $\mathrm{min} \cdot \mathrm{ng} / \mathrm{mL}$ & $468027.55 \pm 210732.88$ \\
$\mathrm{~V}_{\mathrm{d}}$ & $\mathrm{mL} / \mathrm{kg}$ & $34474.41 \pm 17163.13$ \\
$\mathrm{CL}$ & $\mathrm{mL} / \mathrm{min} / \mathrm{kg}$ & $48.59 \pm 16.44$ \\
\hline $\mathrm{MRT}$ & $\mathrm{min}$ & $135.56 \pm 32.22$ \\
\hline
\end{tabular}

FIGURE 6 Pharmacokinetic evaluation of TA in vivo . (a) Pharmacokinetic profile of TA after intraperitoneal (i.p. group) or intravenous (i.v. group) injection in rats. Data are shown as means \pm SEM (n [?] 5). (b) Tissue distribution of TA after intraperitoneal injection in rats. Data are shown as means +- SEM (n $=5)$. (c) Pharmacokinetic parameters of TA after intravenous injection in rats $(\mathrm{n}=6)$. Data are shown as means +- SEM $(\mathrm{n}=6)$. 


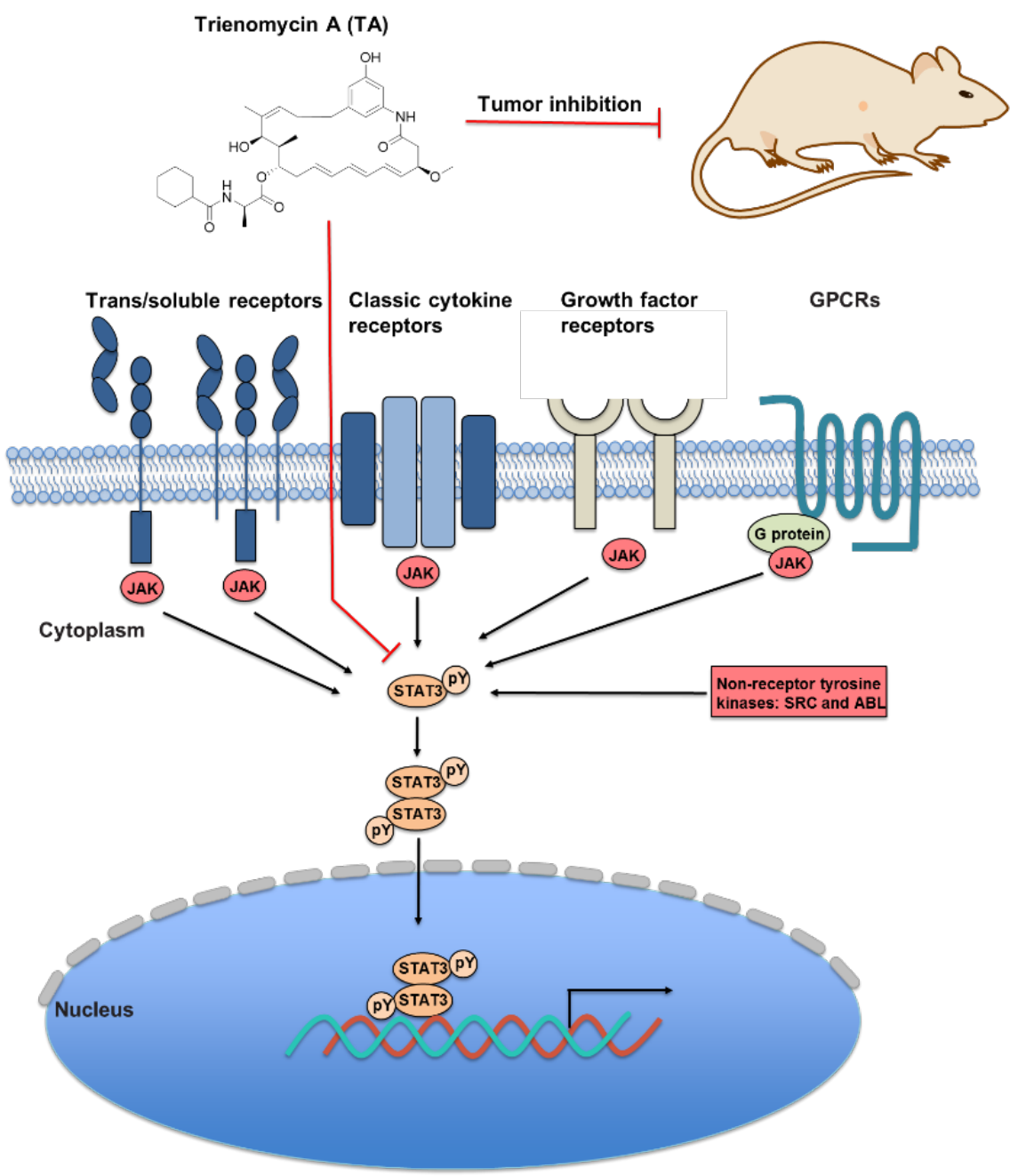

FIGURE 7 Schematic diagram illustrating the mechanism of inhibiting the STAT3 pathway by TA. Cytokines, growth factors and peptide hormones are able to activate the STAT3 signaling pathway. STAT3 is phosphorylated on tyrosine 705 by JAKs and non-receptor tyrosine kinases including SRC and ABL, resulting in STAT3 dimerization and nuclear translocation, followed by binding to palindromic sequences in the genome to initiate the transcription of target genes. In the process, TA directly binds to STAT3 and inhibits STAT3 (Tyr705) phosphorylation, leading to the inhibition of the STAT3 pathway. 